\title{
Multigrid schemes for high order discretizations of hyperbolic problems
}

\author{
Andrea A. Ruggiu \& Jan Nordström \\ Department of Mathematics, Computational Mathematics, Linköping University, \\ SE-581 83 Linköping, Sweden
}

\begin{abstract}
Total variation diminishing multigrid methods have been developed for first order accurate discretizations of hyperbolic conservation laws. This technique is based on a so-called upwind biased residual interpolation and allows for algorithms devoid of spurious numerical oscillations in the transient phase.

In this paper, we justify the introduction of such prolongation and restriction operators by rewriting the algorithm in a matrix-vector notation. This perspective sheds new light on multigrid procedures for hyperbolic problems and provides a direct extension for high order accurate difference approximations. The new multigrid procedure is presented, advantages and disadvantages are discussed and numerical experiments are performed.
\end{abstract}

\section{Introduction}

Multigrid is a convergence acceleration technique originally designed to solve elliptic partial differential equations. ${ }^{1}$ For these problems, it was observed that iterative methods were only damping highly oscillating error modes fast. On the other hand, the low frequency errors could be efficiently flattened by means of grid coarsening, since they were seen as high frequency modes on coarser grids. The main idea behind multigrid techniques is to complement the two effects in order to rapidly get the solution on the finest grid. ${ }^{2,3}$

More recently, multigrid methods were successfully modified to find first order accurate steady state solutions of hyperbolic conservation laws. ${ }^{4,5}$ For these problems, the low frequency errors can possibly be rapidly expelled from the domain by grid coarsening, since coarser grids have less severe stability restrictions on time steps. The new $L$-multigrid procedure allow for $2^{L}$ times faster wave propagation by ensuring that the Total Variation Diminishing (TVD) property is preserved. In the Total Variation Diminishing Multi-Grid scheme (TVD-MG), the residual restriction is performed using an upwind biased interpolation rather than the classical linear one, and this also guarantees convergence of the iterative procedure. However, a significant drawback of the TVD-MG is the intrinsical need for first order accurate space discretizations.

In this article, we will replicate and extend the previously mentioned low order TVD-MG method to upwind Summation-By-Parts (SBP) based high-order finite difference methods ${ }^{6,7}$ for linear hyperbolic problems. The crucial role played by the upwind biased interpolation in the TVD-MG will be investigated from a matrix-vector perspective, leading to a direct extension of the residual restriction to higher order approximations. In our approach, the wave propagation for high-order discretizations on a fine grid is complemented with the TVD-MG for first order schemes on coarse grids. New restriction operators that are needed to couple the high order discretization on the fine grid with the first order scheme on the second finest grid will be presented. We will 
also show how the new residual restriction operators developed for scalar advection problems can be used to accelerate the wave propagation for linear hyperbolic systems.

The article is structured as follows: in Section II we introduce a new first order upwind SBP discretization for one-dimensional scalar advection problems. A multigrid technique for such approximations, inspired by the TVD-MG, is presented in Section III. In this section we also revisit the upwind-biased residual restriction needed for convergence acceleration from a matrix-vector point of view. The extension to higher order discretizations is given in Section IV. Several modifications needed to implement the multigrid algorithm for hyperbolic systems such as the linear compressible Euler equations are discussed in Section V. Finally, conclusions are drawn in Section VI.

\section{Upwind discretization of the advection equation}

Consider the linear wave propagation problem

$$
\begin{aligned}
u_{t}+u_{x} & =f(x), & & 0<x<1, \quad t>0, \\
u(0, t) & =g, & & t>0, \\
u(x, 0) & =u^{0}(x), & & 0<x<1,
\end{aligned}
$$

where $f$ and $g$ are known data independent of time. A semi-discretization of (1) can be written by using SBP operators on upwind form. ${ }^{7}$

Definition II.1. The difference operators $D_{+}=P^{-1}\left(Q_{+}+\frac{B}{2}\right)$ and $D_{-}=P^{-1}\left(Q_{-}+\frac{B}{2}\right)$, with $B=\operatorname{diag}([-1,0, \ldots, 0,1])$, are said to be pth order diagonal-norm upwind SBP operators for the first derivative if

i) $D_{+}\left(D_{-}\right)$is pth and $\lfloor p / 2\rfloor$ th order accurate in the interior and at the left (right) boundary, respectively;

ii) the diagonal matrix $P$ defines a discrete norm;

iii) $Q_{+}+Q_{-}^{T}=0$;

iv) $Q_{+}+Q_{+}^{T}\left(Q_{-}+Q_{-}^{T}\right)$ is positive (negative) semi-definite.

Consider an equidistant grid $\Omega_{1}=\left\{x_{j}=j \Delta x, j=0,1,2, \ldots, N\right\}$ on $[0,1]$, with $N \Delta x=1$. The space discretization of (1) with upwind SBP operators reads

$$
\begin{aligned}
\boldsymbol{U}_{t}+D_{+} \boldsymbol{U} & =\boldsymbol{f}-P^{-1}\left(U_{0}-g\right) \boldsymbol{e}_{0}, \quad t>0 \\
\boldsymbol{U}(0) & =\boldsymbol{U}^{0},
\end{aligned}
$$

where the boundary condition is weakly imposed with a Simultaneous-Approximation-Term (SAT). ${ }^{6}$ In (2), the vector $\boldsymbol{U}=\left(U_{0}, U_{1}, \ldots, U_{N}\right)^{T}$ indicates the approximate solution with $U_{j}(t) \approx u\left(x_{j}, t\right)$ and $\boldsymbol{f}=\left(f\left(x_{0}\right), f\left(x_{1}\right), \ldots, f\left(x_{N}\right)\right)^{T}$. Moreover, we have used $\boldsymbol{e}_{0}=(1,0, \ldots, 0)^{T}$. Note that the positive variant of the SBP upwind operator has been used, since the solution of (1) is a wave propagating towards the right boundary.

As for the usual centered SBP operators, ${ }^{6,8}$ it is straightforward to show that (2) leads to stability. ${ }^{7}$ Indeed, by letting $\mathbf{f}=\mathbf{0}$ and applying the energy method, i.e. multiplying (2) from the left by $\boldsymbol{U}^{T} P$ and adding the transpose of the resulting expression, we get

$$
\frac{\mathrm{d}}{\mathrm{d} t}\|\mathbf{U}\|_{P}^{2}=g^{2}-U_{N}^{2}-\left(U_{0}-g\right)^{2}-\mathbf{U}^{T}\left(Q_{+}+Q_{+}^{T}\right) \mathbf{U} .
$$

Since $Q_{+}+Q_{+}^{T}$ is positive semi-definite, $\|\mathbf{U}\|_{P}=\mathbf{U}^{T} P \mathbf{U}$ will be bounded by data. 


\section{II.A. New first order upwind SBP operators}

The upwind SBP operators in Definition II.1 were derived from second up to ninth order. ${ }^{7}$ However, in the present study we will also need first order upwind discretizations satisfying the SBP property in order to replicate and extend the TVD-MG technique. ${ }^{4,5}$ Such operators (for the positive variant) are given by

$$
D_{+}=\frac{1}{\Delta x}\left[\begin{array}{cccc}
0 & & & \\
-1 & 1 & & \\
& \ddots & \ddots & \\
& & -1 & 1
\end{array}\right], \quad P=\Delta x \cdot \operatorname{diag}(1, \ldots, 1), \quad Q_{+}=\left[\begin{array}{cccc}
\frac{1}{2} & & & \\
-1 & 1 & & \\
& \ddots & \ddots & \\
& & -1 & \frac{1}{2}
\end{array}\right] .
$$

The operator $D_{+}$is first order accurate in all the nodes with the exception of the left boundary closure, where the order of accuracy drops to zero. The particular structure of $D_{+}$makes it easy to compute the steady-state solution of (2) as

$$
U_{i}=g_{0}+\Delta x \sum_{k=0}^{i} f_{k}
$$

which is the discrete analogous of the steady-state solution of (1)

$$
u(x)=g_{0}+\int_{0}^{x} f(\xi) \mathrm{d} \xi .
$$

Remark II.2. The matrix $P$ in (3) does not represent a consistent quadrature formula, namely it does not correctly integrate constant grid functions. Consistency can be restored by relaxing the constraint on the matrix $P$ defining a discrete norm. In particular, the modified matrix $P_{+}=\Delta x$. $\operatorname{diag}(0,1, \ldots, 1)$ exactly integrates constants and leads to the same $D_{+}$as in $(3)$, and defines a seminorm. However, this matrix can only be used for the positive variant $D_{+}$, since the corresponding negative variant $D_{-}$requires $P_{-}=\Delta x \cdot \operatorname{diag}(1, \ldots, 1,0)$. Hence, to make the matrix $P$ both strictly positive definite and equal for the two variants, the consistency requirement is relaxed. This lack of consistency is in line with the previously derived upwind SBP operators: indeed, the norm $P$ of a pth order SBP upwind first derivative operator exactly integrates only $(p-2)$ th degree polynomials, if $p$ is odd.

\section{II.B. Convergence to steady-state for single grid methods}

The steady state solution (4) can also be found by discretizing (2) in time and computing an approximation of $\boldsymbol{U}(t)$ for large $t$. By marching in time with Euler Forward (EF) we get

$$
\boldsymbol{U}^{n+1}=\boldsymbol{U}^{n}-\Delta t\left(D_{+}+P^{-1} \boldsymbol{e}_{0} \boldsymbol{e}_{0}^{T}\right) \boldsymbol{U}^{n}+\Delta t \boldsymbol{F},
$$

where $\boldsymbol{F}=\boldsymbol{f}+g(\Delta x)^{-1} \boldsymbol{e}_{0}$ and the superscript $n$ denotes the approximation at time $t^{n}=n \Delta t$. The discretization (6) converges to steady-state without spurious oscillations if $\Delta t \leq \Delta x$. Moreover, it can be recast in compact form by introducing

$$
L_{1}=D_{+}+P^{-1} e_{0} \boldsymbol{e}_{0}^{T}=\frac{1}{\Delta x}\left[\begin{array}{cccc}
1 & & \\
-1 & 1 & \\
& \ddots & \ddots & \\
& & -1 & 1
\end{array}\right]
$$


and

$$
S_{1}=I_{1}-\Delta t L_{1}=\left[\begin{array}{cccc}
1-\lambda & & & \\
\lambda & 1-\lambda & & \\
& \ddots & \ddots & \\
& & \lambda & 1-\lambda
\end{array}\right] .
$$

In (7),(8), $I_{1}$ is the identity matrix, $\lambda=\Delta t / \Delta x$ denotes the CFL number and the subscript 1 is used to denote quantities on the grid $\Omega_{1}$. With this notation, the method (6) can be rewritten as

$$
\boldsymbol{U}^{n+1}=S_{1} \boldsymbol{U}^{n}+\left(I_{1}-S_{1}\right) L_{1}^{-1} \boldsymbol{F} .
$$

A sufficient condition for the convergence of (9) is that the eigenvalues of $L_{1}$ lie on the right-half of the complex plane. ${ }^{9,10}$ Additionally, the spectral radius of $S_{1}$, i.e. the maximum among the absolute values of its eigenvalues, must be less than one for convergence.

Remark II.3. The invertibility of $L_{1}$ can be shown for pseudo-spectral approximations, ${ }^{9}$ but not in general for discretizations based on finite-difference methods. ${ }^{10}$ The 1 st and 2 nd order upwind SBP operators lead to matrices $L_{1}$ with triangular and block-triangular structure, respectively, for which invertibility follows in a straightforward way. However, it is presently not known how to extend this result to higher order finite difference approximations.

For a first order upwind operator $D_{+}$and $\lambda=1$, the convergence to steady-state is achieved in $N+1$ iterations, since

$$
U_{0}^{n+1}=g_{0}+\Delta x f_{0}, \quad U_{j}^{n+1}=U_{j-1}^{n}+\Delta x f_{j}, \quad j=1, \ldots, N
$$

yield

$$
U_{N}^{N+1}=U_{N-1}^{N}+\Delta x f_{N}=U_{N-2}^{N-1}+\Delta x\left(f_{N-1}+f_{N}\right)=\ldots=g_{0}+\Delta x \sum_{k=0}^{N} f_{k} .
$$

Other explicit schemes can of course also be used for the time advancement of (2). For example, the fourth order Runge-Kutta (RK4) scheme leads to the iterative procedure (9) with

$$
S_{1}=I_{1}-\Delta t L_{1}+\frac{1}{2}\left(\Delta t L_{1}\right)^{2}-\frac{1}{6}\left(\Delta t L_{1}\right)^{3}+\frac{1}{24}\left(\Delta t L_{1}\right)^{4} .
$$

Although also this iterative method converges to the steady-state solution, the EF time marching for first order discretizations leads to faster convergence. In Figure 1 the approximate solution of (2) with $\mathbf{U}^{0}=(1, \ldots, 1)^{T}, f=0$ and $g=0$ is displayed at $t=0.5$ for both procedures with $\lambda=0.01$. The RK4 method damps the initial discontinuity, and thereby slows down the convergence to the steady-state solution $\mathbf{U}=(0, \ldots, 0)^{T}$.

Using (10) leads to a transient phase consisting of both a convective part and a damping part. The latter does not arise with EF, as can be seen in Figure 2. Purely convective convergence is a distinctive feature of first order upwind space discretizations using EF. On the other hand, using EF is inappropriate in combination with higher order discretizations due to its poor stability properties. For these cases, (10) is preferred.

\section{Convergence acceleration for first order upwind schemes}

Consider the discrete problem (6), which converges to the steady-state solution $\mathbf{U}=L_{1}^{-1} \mathbf{F}_{1}$ in $N+1$ iterations. The wave propagation speed can be increased by using the following two-level algorithm, which is inspired by the TVD-MG: ${ }^{4,5}$

Fine grid smoothing: $\quad \overline{\boldsymbol{U}}^{(1)}=S_{1} \boldsymbol{U}^{n}+\left(I_{1}-S_{1}\right) L_{1}^{-1} \boldsymbol{F}_{1} ;$ 

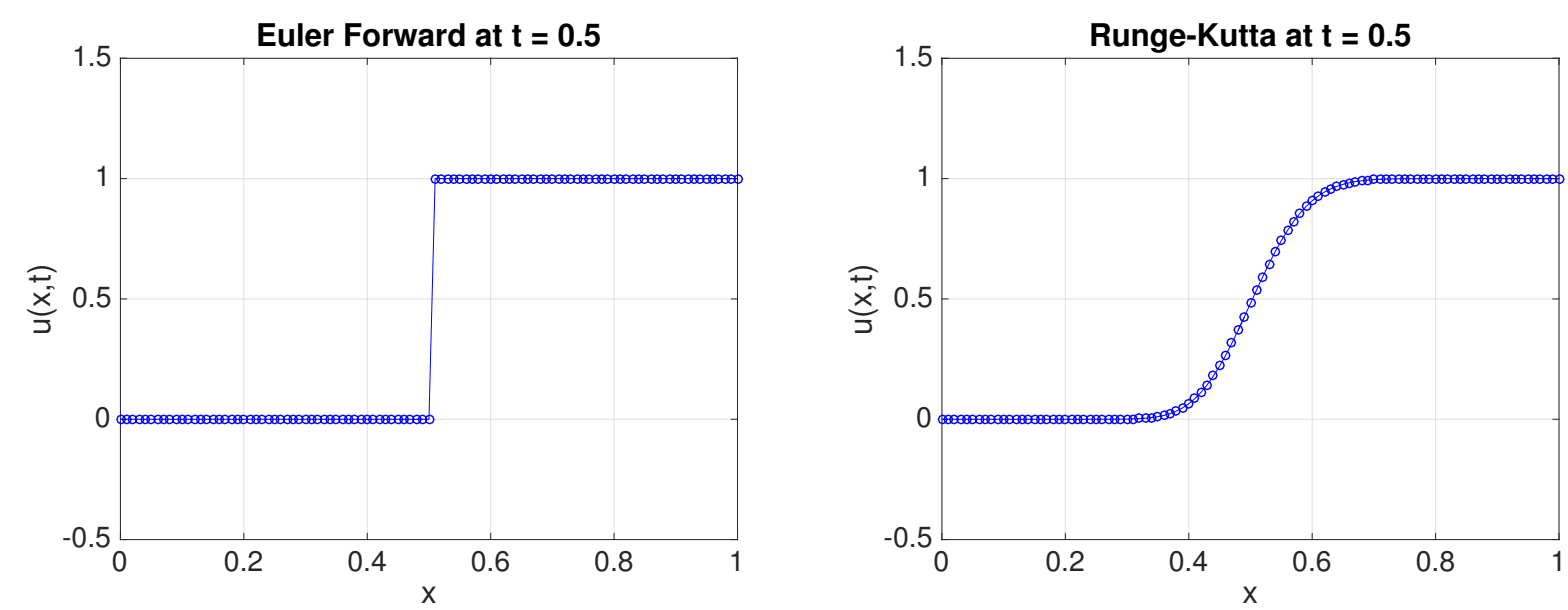

Figure 1. Approximate solution of (2) with $f=0, g=0, \mathbf{U}^{(0)}=(1, \ldots, 1)^{T}$ and the first order space discretization. The solution is shown at $t=0.5$ for EF (left) and RK4 (right) time integrators with $\lambda=0.01$.
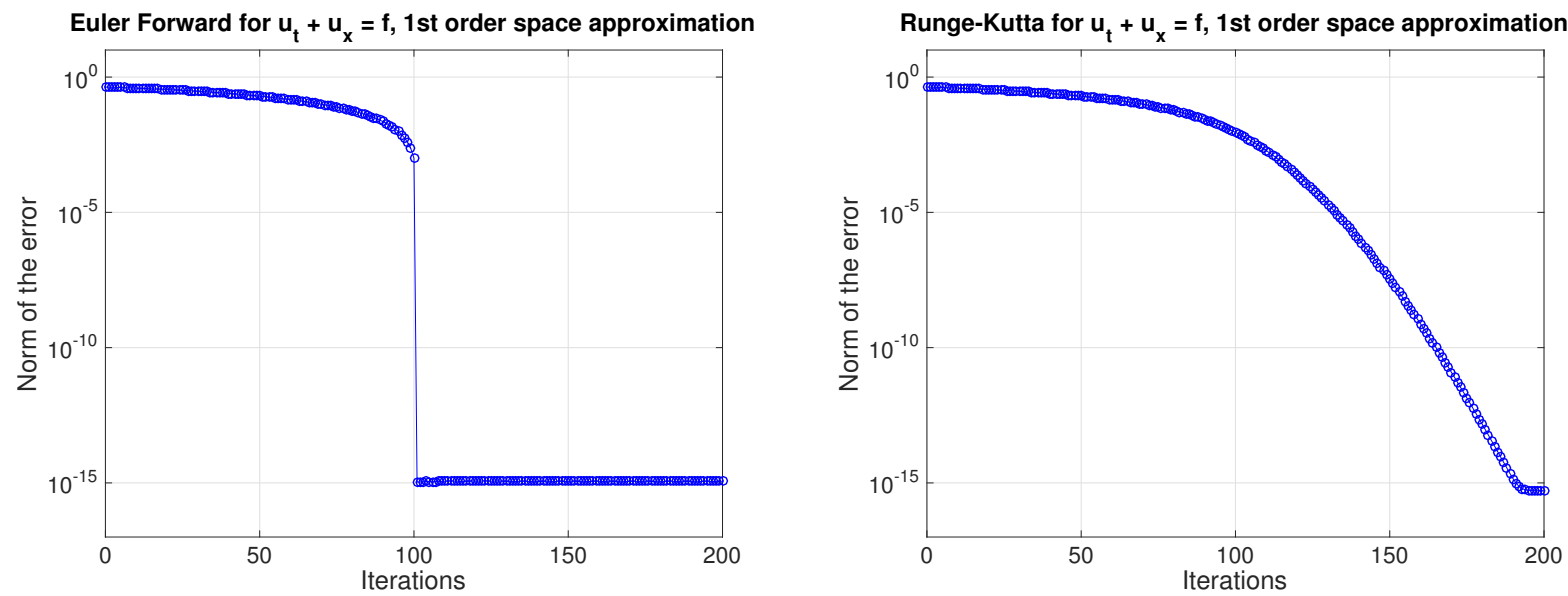

Figure 2. Convergence plots for the problem $(2)$ with $f(x)=\cos (x)-x \sin (x), g=1, \mathbf{U}^{(0)}=(1, \ldots, 1)^{T}$. The $P$-norm of the error with respect to the steady-state solution is shown for both EF (left) and RK4 (right) time-marching schemes with $\lambda=0.01$.

Fine grid residual: $\quad \overline{\boldsymbol{r}}^{(1)}=L_{1} \overline{\boldsymbol{U}}^{(1)}-\boldsymbol{F}_{1}$;

Solution injection: $\quad \boldsymbol{U}^{(2)}=\mathcal{R}_{u} \overline{\boldsymbol{U}}^{(1)}$;

Coarse grid residual: $\quad \boldsymbol{F}_{2}=L_{2} \boldsymbol{U}^{(2)}-I_{r} \overline{\boldsymbol{r}}^{(1)}$;

Coarse grid evolution: $\quad \overline{\boldsymbol{U}}^{(2)}=S_{2} \boldsymbol{U}^{(2)}+\left(I_{2}-S_{2}\right) L_{2}^{-1} \boldsymbol{F}_{2}$;

Fine grid update: $\quad \boldsymbol{U}^{n+1}=\overline{\boldsymbol{U}}^{(1)}+I_{p}^{I}\left(\overline{\boldsymbol{U}}^{(2)}-\mathcal{R}_{u} \overline{\boldsymbol{U}}^{(1)}\right)+I_{p}^{E}\left(\overline{\boldsymbol{U}}^{(2)}-\mathcal{R}_{u} \boldsymbol{U}^{n}\right)$.

Quantities on the fine and coarse grid are indicated with superscripts 1, 2, respectively.

In (11) we have introduced:

- A coarse grid $\Omega_{2}=\left\{x_{j}^{(2)}=2 j \Delta x^{(1)}=j \Delta x^{(2)}, j=0,2, \ldots, N\right\} \subset[0,1]$;

- A restriction operator of injection type $\mathcal{R}_{u}$ such that $\left(\mathcal{R}_{u} \boldsymbol{v}^{(1)}\right)_{j}=v_{j}^{(2)}$ for $j=0,2, \ldots, N$; 
- A residual restriction operator $I_{r}$ which conveys the information from $\Omega_{1}$ to $\Omega_{2}$,

$$
\left(I_{r} \boldsymbol{v}^{(1)}\right)_{j}= \begin{cases}\frac{1}{2} v_{0}^{(1)}, & j=0, \\ \frac{1}{2}\left(v_{j}^{(1)}+v_{j-1}^{(1)}\right), & j=2,4, \ldots, N,\end{cases}
$$

which is upwind-biased ${ }^{4,5}$ at interior nodes and inconsistent at the left boundary node. This somewhat odd choice will be explained later. See Figure 3 for details;

- A space discretization $L_{2}=D_{+, 2}+P_{2}^{-1} \mathbf{e}_{0,2} \mathbf{e}_{0,2}^{T}$ and a smoother $S_{2}=I_{2}-\Delta t_{2} L_{2}$ on the coarse grid $\Omega_{2}$ which are counterparts to the operators $L_{1}$ and $S_{1}$ in (7),(8) on $\Omega_{1}$;

- The prolongation operator $I_{p}=I_{p}^{I}+I_{p}^{E}$ in the fine grid update step $^{11}$ has been split into two contributions: $I_{p}^{I}$ for the nodes included on the coarse grid and $I_{p}^{E}$ for the other nodes, see Figure 3. In particular,

$$
\begin{aligned}
& \left(I_{p}^{I} \boldsymbol{v}^{(2)}\right)_{j}= \begin{cases}v_{j}^{(2)}, & j=0,2,4, \ldots, N, \\
0, & j=1,3,5, \ldots, N-1,\end{cases} \\
& \left(I_{p}^{E} \boldsymbol{v}^{(2)}\right)_{j}= \begin{cases}0, & j=0,2,4, \ldots, N, \\
v_{j-1}^{(2)}, & j=1,3,5, \ldots, N-1,\end{cases}
\end{aligned}
$$

are introduced to avoid overshoots in the transient phase, and preserve the TVD property. ${ }^{4}$
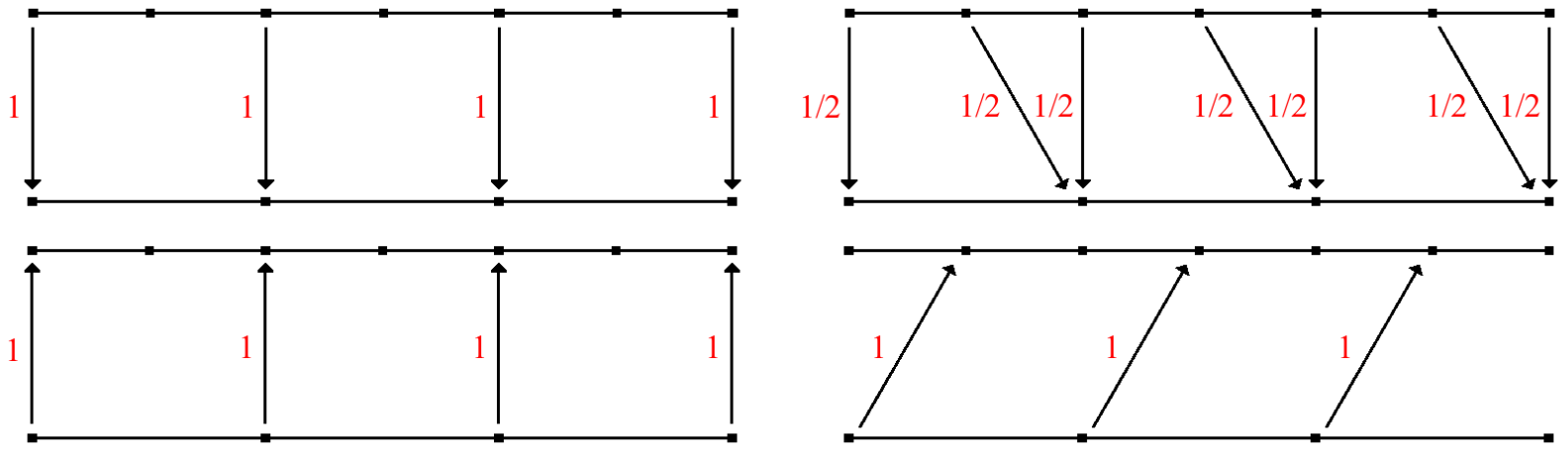

Figure 3. The interpolation operators used in the two-level algorithm (11) are the injection operator $\mathcal{R}_{u}$ (top left), the restriction operator $I_{r}$ (top right), the prolongation operator for the included nodes $I_{p}^{I}$ (bottom left) and the prolongation operator for the excluded nodes $I_{p}^{E}$ (bottom right). Note that $I_{r}$ is inconsistent at the left boundary node.

In this algorithm, the residual problem $L_{1} \mathbf{E}^{(1)}=L_{1} \overline{\boldsymbol{U}}^{(1)}-\mathbf{F}_{1}=\overline{\mathbf{r}}^{(1)}$, with $\mathbf{E}^{(1)}=\overline{\mathbf{U}}^{(1)}-\mathbf{U}$, is restricted onto the coarse-grid by means of $I_{r}$. Since the resulting problem $I_{r} L_{1} \mathbf{E}^{(1)}=I_{r} \overline{\mathbf{r}}^{(1)}$ contains the rectangular matrix $I_{r} L_{1}$, it is substituted with a problem on $\Omega_{2}$ involving the square matrix $L_{2}$. In particular, by assuming that

$$
I_{r} L_{1} \mathbf{E}^{(1)} \approx L_{2} \mathcal{R}_{u} \mathbf{E}^{(1)}
$$

the problem is substituted with $L_{2} \mathcal{R}_{u} \mathbf{E}^{(1)}=I_{r} \overline{\mathbf{r}}^{(1)}$, or equivalently

$$
L_{2} \mathcal{R}_{u} \mathbf{U}=L_{2} \mathcal{R}_{u} \overline{\mathbf{U}}^{(1)}-I_{r} \overline{\mathbf{r}}^{(1)}=: \mathbf{F}_{2}
$$


As a consequence, the augmented problem in time $\mathbf{V}_{t}+L_{2} \mathbf{V}=\mathbf{F}_{2}$ converges towards the steadystate solution $\mathcal{R}_{u} \mathbf{U}$. After a number of smoothing steps, this problem provides an approximation $\overline{\mathbf{U}}^{(2)}$ which is used in the fine grid update for a new approximate solution $\mathbf{U}^{(n+1)}$ on the fine grid.

The convergence of the algorithm in (11) can be studied by rewriting it in compact form as

$$
\boldsymbol{U}^{n+1}=M \boldsymbol{U}^{n}+\left(I_{1}-M\right) L_{1}^{-1} \boldsymbol{F}_{1}
$$

where

$$
M=\left(I_{1}-I_{p}\left(I_{2}-S_{2}\right) L_{2}^{-1} I_{r} L_{1}\right) S_{1}-I_{p}^{E} \mathcal{R}_{u}\left(I_{1}-S_{1}\right)
$$

is the multigrid iteration matrix.

Remark III.1. The only formal difference between the algorithm in (11) and the conventional two-grid procedure ${ }^{11}$ is in the fine-grid update step. Indeed, by changing that to the usual

$$
\boldsymbol{U}^{n+1}=\overline{\boldsymbol{U}}^{(1)}+I_{p}\left(\overline{\boldsymbol{U}}^{(2)}-\mathcal{R}_{u} \overline{\boldsymbol{U}}^{(1)}\right)
$$

the resulting multigrid iteration matrix for the two approaches has the same structure, i.e.

$$
M=\left(I_{1}-I_{p}\left(I_{2}-S_{2}\right) L_{2}^{-1} I_{r} L_{1}\right) S_{1} .
$$

Using the first order upwind scheme discretized in time with EF (6) for both the fine- and coarse-grid discretizations, $M$ becomes

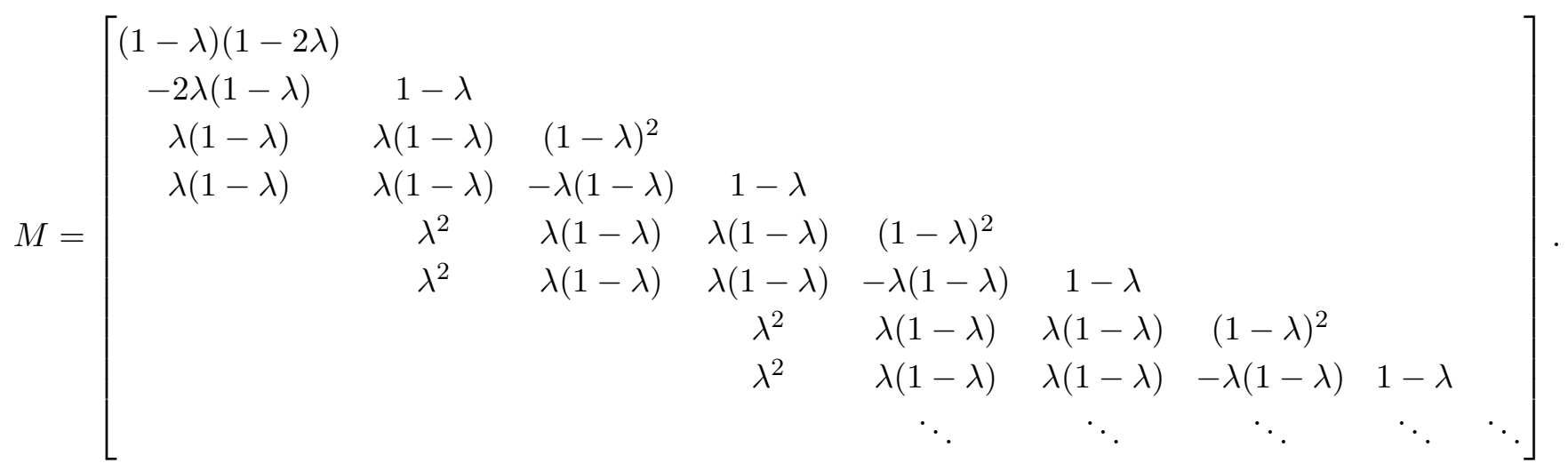

It is easy to verify that the multigrid iteration scheme (15) converges for $\lambda \leq 1$, since the eigenvalues of $M$ are given by its diagonal entries. Moreover, for the specific choice $\lambda=1$, convergence is achieved in exactly $N / 4+1$ iterations. It is also possible to recursively apply grid coarsening to speed up the procedure: for $L$ grids, the multigrid algorithm can be written as

$$
\begin{aligned}
& k=1 ; \\
& \mathbf{U}^{(1)}=\mathbf{U}^{n} ; \\
& \overline{\mathbf{U}}^{(1)}=S_{1} \mathbf{U}^{(1)}+\left(I_{1}-S_{1}\right) L_{1}^{-1} \mathbf{F}_{1} ;
\end{aligned}
$$

while $k<L$

$$
\begin{aligned}
& \overline{\boldsymbol{r}}^{(k)}=L_{k} \overline{\boldsymbol{U}}^{(k)}-\boldsymbol{F}_{k} ; \\
& \boldsymbol{U}^{(k+1)}=\mathcal{R}_{u}^{(k \rightarrow k+1)} \overline{\boldsymbol{U}}^{(k)} ; \\
& \boldsymbol{F}_{k+1}=L_{k+1} \boldsymbol{U}^{(k+1)}-I_{r}^{(k \rightarrow k+1)} \overline{\boldsymbol{r}}^{(k)} ; \\
& \overline{\boldsymbol{U}}^{(k+1)}=S_{k+1} \boldsymbol{U}^{(k+1)}+\left(I_{k+1}-S_{k+1}\right) L_{k+1}^{-1} \boldsymbol{F}_{k+1} ;
\end{aligned}
$$




$$
k=k+1
$$

end

$$
\widetilde{\mathbf{U}}^{(L)}=\overline{\mathbf{U}}^{(L)} \text {; }
$$

while $k>1$

$$
\begin{aligned}
& \widetilde{\mathbf{U}}^{(k-1)}=\overline{\mathbf{U}}^{(k-1)}+I_{p}^{I,(k \rightarrow k-1)}\left(\widetilde{\boldsymbol{U}}^{(k)}-\mathcal{R}_{u}^{(k-1 \rightarrow k)} \overline{\boldsymbol{U}}^{(k-1)}\right) \\
& +I_{p}^{E,(k \rightarrow k-1)}\left(\widetilde{\boldsymbol{U}}^{(k)}-\mathcal{R}_{u}^{(k-1 \rightarrow k)} \boldsymbol{U}^{(k-1)}\right) ; \\
& k=k-1 ;
\end{aligned}
$$

end

$$
\mathbf{U}^{n+1}=\widetilde{\mathbf{U}}^{(1)} \text {. }
$$

In (17), the superscript $(m \rightarrow n)$ indicates that the interpolation operator transfers information from the grid $m$ to the grid $n$. For notation simplicity, this superscript is neglected when considering the finest and the second finest grid.

The multigrid algorithm (17) converges in $N / 2^{L}+1$ iterations (see Figure 4). These results are
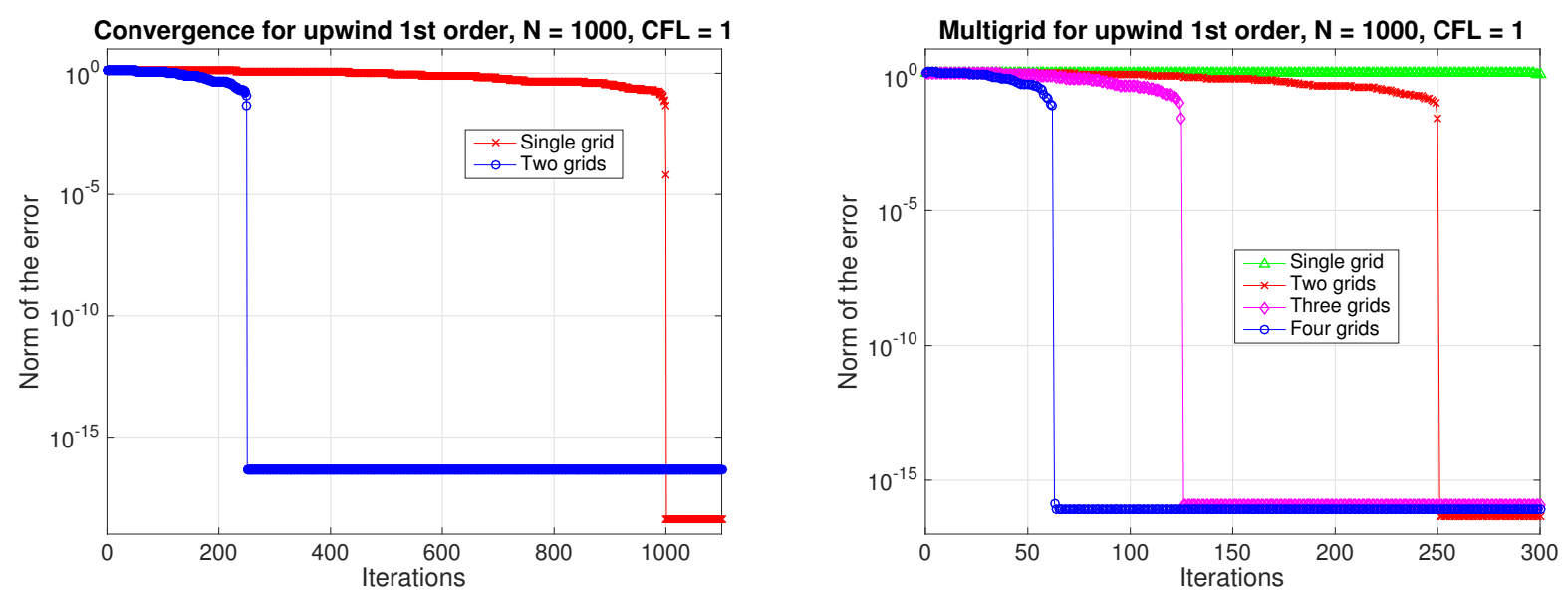

Figure 4. The left figure shows the $P$-norm of the error for a first order accurate single- and two-grid scheme with $N=1000$ and $\lambda=1$. We have used the manufactured solution $u_{s}(x)=$ $e^{-x}(\cos (10 \pi x)+\cos (2 \pi x))$ and a compatible random initial data. The convergence of the multigrid procedure is displayed in the right figure.

in line with the ones obtained with the TVD-MG ${ }^{4}$ where the boundary conditions were strongly imposed.

Remark III.2. For all the numerical experiments in Section III and IV we have used the manufactured solution $u_{s}(x)=e^{-x}(\cos (10 \pi x)+\cos (2 \pi x))$ and a random initial data compatible with the boundary condition.

Remark III.3. The multigrid algorithm (17) is referred to as a multiplicative scheme. ${ }^{4,5} A$ so-called additive scheme can be obtained by replacing $\boldsymbol{U}^{(k+1)}=\mathcal{R}_{u}^{(k \rightarrow k+1)} \overline{\boldsymbol{U}}^{(k)}$ with $\boldsymbol{U}^{(k+1)}=$ $\mathcal{R}_{u}^{(k \rightarrow k+1)} \boldsymbol{U}^{(k)}$. However, the resulting algorithm turns out to be slower than the multiplicative version and hence only (17) will be considered in the following. 


\section{III.A. Initial modifications for higher order discretizations}

The matrix-vector notation introduced in $(11),(17)$ can be used to generalize and adapt the multigrid algorithm for higher order discretizations. As a first attempt, we substitute $D_{+}$in (2) with the SBP upwind operators of 3rd, 4th, 5th and 6th order for all the grids involved. For all the levels, the RK4 time integrator with $\lambda=0.5$ is used. The convergence results in Figure 5 show that the multigrid procedure either becomes ineffective or ceases to converge for more than 2 grid levels.
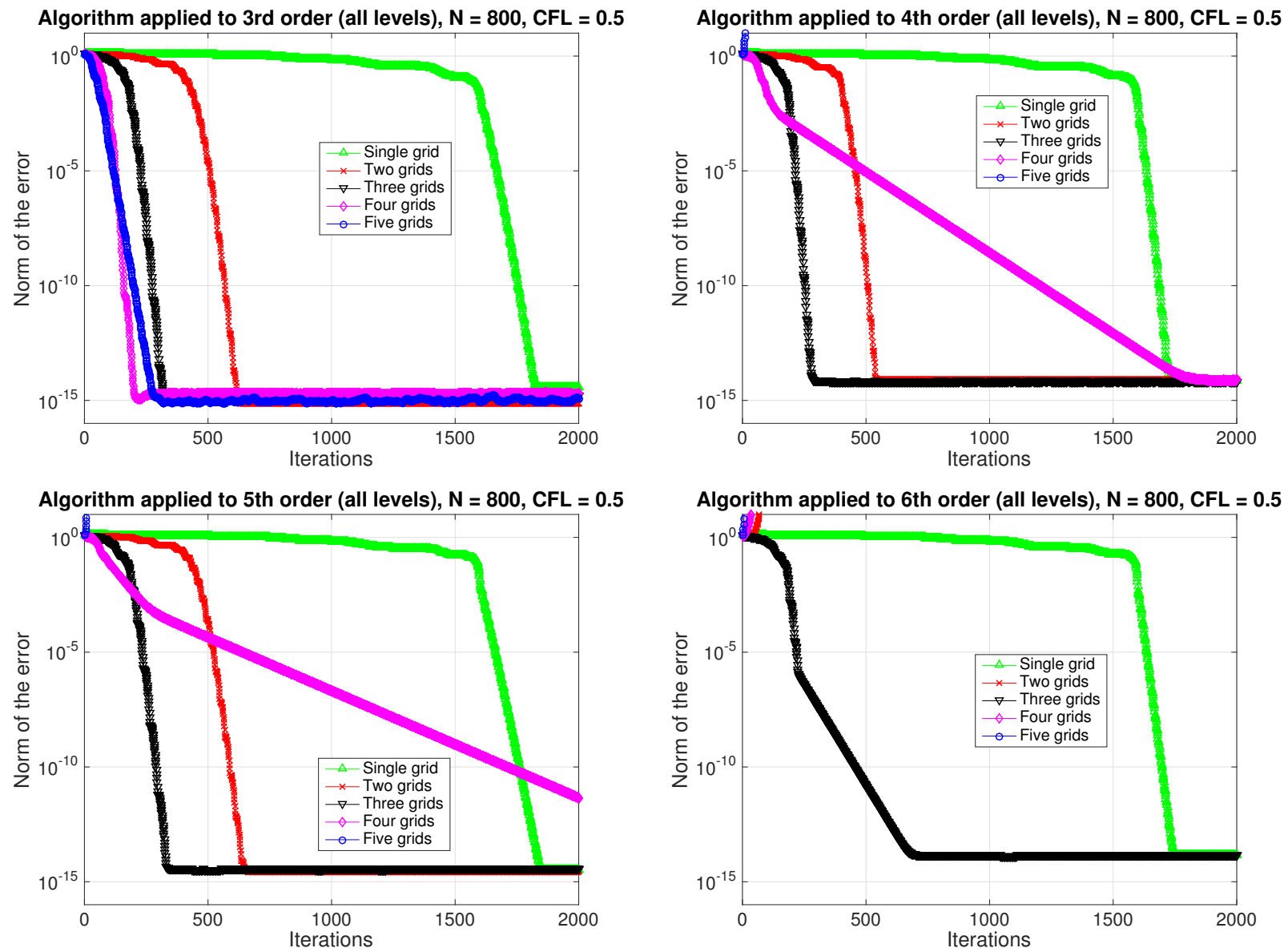

Figure 5. Convergence plots for the multigrid algorithm in (17) with 3rd (top left), 4th (top right), 5th (bottom left), 6th (bottom right) order discretizations for all grids. RK4 time marching with $\lambda=0.5$ was used on each grid.

A second attempt to extend the multigrid procedure to higher order discretizations was made by recalling that the accuracy of the steady state solution does not depend on the accuracy on the coarse grids. Thus, we kept a first order upwind discretization for $L_{k}, k=2,3, \ldots$. The convergence plots for the multigrid procedure with high order discretization on the fine grid and first order discretization on coarser grids are shown in Figure 6. Once again, the multigrid algorithm does not produce satisfactory results, at least not for orders of accuracy higher than 3 .

The outcome of these numerical experiments shows that an extension to higher order discretizations requires a deeper understanding of the interpolation operators and further analysis. 

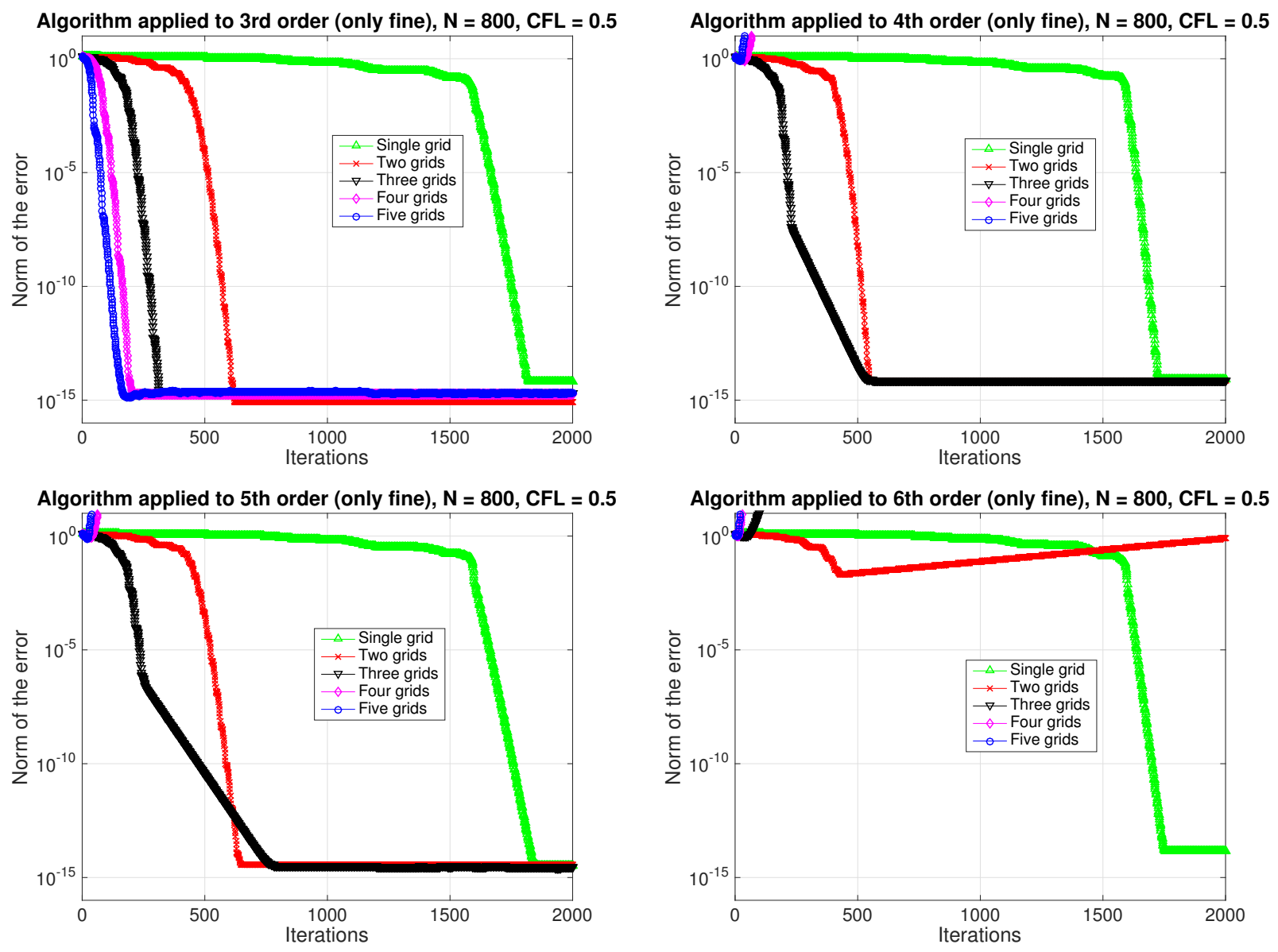

Figure 6. Convergence plots for the multigrid algorithm in (17) with 3rd (top left), 4th (top right), 5th (bottom left), 6th (bottom right) order discretization for the fine grid and first order discretization for the coarser grids. RK4 time marching with $\lambda=0.5$ was used on each grid for wave propagation.

\section{III.B. Revisiting the upwind-biased residual restriction}

Due to the pointwise notation and strong imposition of the boundary conditions used for the TVD$\mathrm{MG}$, the upwind biased operator $I_{r}(12)$ was previously presented only for the interior nodes of the discretization. In that case, it can be shown that this residual restriction, for $\mathbf{F}_{1}=\mathbf{0}$, led to ${ }^{4}$

$$
\begin{aligned}
\left(\mathbf{F}_{2}\right)_{j} & =\frac{1}{\Delta x^{(2)}}\left(U_{j}^{(2)}-U_{j-2}^{(2)}\right)-\frac{1}{2}\left(\bar{r}_{j}^{(1)}+\bar{r}_{j-1}^{(1)}\right) \\
& =\frac{1}{\Delta x^{(2)}}\left(\bar{U}_{j}^{(1)}-\bar{U}_{j-2}^{(1)}\right)-\frac{1}{2 \Delta x^{(1)}}\left[\left(\bar{U}_{j}^{(1)}-\bar{U}_{j-1}^{(1)}\right)+\left(\bar{U}_{j-1}^{(1)}-\bar{U}_{j-2}^{(1)}\right)\right]=0
\end{aligned}
$$

since $\Delta x^{(2)}=2 \Delta x^{(1)}$. On the other hand, the coarse grid residual step of the algorithm (11) can be recast as

$$
\boldsymbol{F}_{2}=L_{2} \boldsymbol{U}^{(2)}-I_{r} \overline{\mathbf{r}}^{(1)}=\left(L_{2} \mathcal{R}_{u}-I_{r} L_{1}\right) \overline{\boldsymbol{U}}^{(1)}+I_{r} \boldsymbol{F}_{1},
$$

in which each step of (18) is mimicked. By comparing (18) and (19), it is clear that the upwind biased restriction operator ${ }^{4,5}$ satisfies $L_{2} \mathcal{R}_{u}=I_{r} L_{1}$, verifying exactly the assumption in (14), on 
the interior nodes of $\Omega_{2}$. To extend this property also to the boundary nodes we compute

$$
I_{r}^{+}=L_{2} \mathcal{R}_{u} L_{1}^{-1}=\left[\begin{array}{ccccccccc}
\frac{1}{2} & & & & & & & \\
& \frac{1}{2} & \frac{1}{2} & & & & & & \\
& & & \frac{1}{2} & \frac{1}{2} & & & & \\
& & & & & \ddots & \ddots & & \\
& & & & & & & \frac{1}{2} & \frac{1}{2}
\end{array}\right],
$$

which explains the choice (12) which is identical to (20). $I_{r}^{+}$in (12),(20) is inconsistent at the left boundary node and matches the previously developed upwind biased restriction on the other nodes. The main advantage with this restriction is that it leads to the following coarse grid evolution step

$$
\overline{\boldsymbol{U}}^{(2)}=S_{2} \boldsymbol{U}^{(2)}+\left(I_{2}-S_{2}\right) L_{2}^{-1} I_{r}^{+} \mathbf{F}_{1}=S_{2}\left(\mathcal{R}_{u} \overline{\mathbf{U}}^{(1)}\right)+\left(I_{2}-S_{2}\right)\left[\mathcal{R}_{u}\left(L_{1}^{-1} \mathbf{F}_{1}\right)\right],
$$

which highlights a clear connection between the fine- and coarse-grid problems.

Remark III.4. Roughly speaking, by satisfying $L_{2} \mathcal{R}_{u}=I_{r}^{+} L_{1}$ we ensure that the coarse grid evolution step involves only unbiased components of the fine grid problem: the coarse grid iterative method takes the injection of the solution of the fine grid smoothing step and converges towards the injection of the exact steady-state solution of the fine grid problem, $L_{1}^{-1} \mathbf{F}_{1}$. In other words, the fine- and coarse-grid problems converge to the same solution on the nodes of $\Omega_{2}$.

The derivation of $I_{r}^{+}$can of course be repeated for propagation problems with left-traveling waves discretized in space with the negative variant of the first order SBP upwind operators. This leads to a residual restriction operator which is rotated with respect to its positive counterpart

$$
I_{r}^{-}=\left[\begin{array}{lllllllll}
\frac{1}{2} & \frac{1}{2} & & & & & & & \\
& & \frac{1}{2} & \frac{1}{2} & & & & & \\
& & & \ddots & \ddots & & & \\
& & & & & \frac{1}{2} & \frac{1}{2} & \\
& & & & & & & \frac{1}{2}
\end{array}\right] .
$$

In the following sections we will use $I_{r}^{+}$and $I_{r}^{-}$for the right- and left-traveling waves, respectively. Similarly, the prolongation operators will be denoted with the superscript + or - depending on the direction of propagation.

\section{Extension to higher orders of accuracy}

To generalize the multigrid procedure for (1) to higher order accuracy, we recall that a unique restriction operator $I_{r}^{+}$such that $L_{2} \mathcal{R}_{u}=I_{r}^{+} L_{1}$ exists if $L_{1}=D_{+}+P^{-1} \mathbf{e}_{0} \mathbf{e}_{0}^{T}$ is invertible. This relation should hold also for the residual restriction between coarser grids. However, since the order of convergence does not depend on the accuracy of the operators on the coarse grids, we choose, as we did in Section III, to use a first order upwind scheme for the coarse grid operators $L_{i}, i=2,3, \ldots$. The obvious advantage is that the residual restriction in (12),(20) automatically satisfies the constraint $L_{k+1} \mathcal{R}_{u}^{(k \rightarrow k+1)}=I_{r}^{+,(k \rightarrow k+1)} L_{k}$ for $k=2,3, \ldots$ In other words, to accelerate the convergence for higher order discretizations we use the already existing TVD-MG for first order schemes $^{4,5}$ on coarse grids. The crucial modification to the former algorithm is the introduction of new residual interpolation operators between the fine and the second finest grids, which depends on the order of accuracy of $L_{1}$. 
Note that by demanding $I_{r}^{+}=L_{2} \mathcal{R}_{u} L_{1}^{-1}$ we can simplify the two-grid matrix $M$ in (16) to

$$
M=\left(I_{1}-I_{p}^{+}\left(I_{2}-S_{2}\right) \mathcal{R}_{u}\right) S_{1}-I_{p}^{E,+} \mathcal{R}_{u}\left(I_{1}-S_{1}\right),
$$

where $I_{p}^{+}=I_{p}^{I}+I_{p}^{E,+}$. This matrix and, in general, the convergence properties of the multigrid scheme are now formally independent of $I_{r}^{+}$. However, to use the algorithm in practice we need to compute $\boldsymbol{F}_{2}=I_{r}^{+} \boldsymbol{F}_{1}$ and hence we still need the restriction operators $I_{r}^{+}$in closed form.

\section{IV.A. Prolongation operators}

Before moving on to convergence acceleration for higher order discretizations, we briefly discuss how to choose the interpolation operator $I_{p}^{+}$in general. Since it was possible to revisit the role of the residual restriction operator $I_{r}^{+}$and prescribe an explicit formula for its computation, one may wonder if this can be done also for the prolongation operator. For first order upwind approximations in space of (1) discretized in time with $\mathrm{EF}$ and $\lambda=1$, the additional property

$$
\left(I_{1}-I_{p}^{I} \mathcal{R}_{u}\right) S_{1}=I_{p}^{E,+} \mathcal{R}_{u}
$$

holds. With (22), the multigrid matrix (21) simplifies to $M=I_{p}^{+} S_{2} \mathcal{R}_{u} S_{1}$. This indicates that for each multigrid cycle, the errors are first propagated by means of the fine grid solver, secondly injected on the coarse grid, next propagated there and finally transferred back onto the fine grid.

Unfortunately, it is not possible to exactly solve (22) for $I_{p}^{E,+}$ for higher order discretizations, since $\mathcal{R}_{u}$ is a rectangular matrix which leads to an overdetermined system. Since we are not able to mimic the behavior of the first order upwind discretization, our analysis will be limited to other classes of prolongation operators. In particular we will consider:

1. The linear prolongation operator $I_{p}^{+}$in (13).

2. The prolongation operator $I_{p}^{+}=I_{p}^{I}+I_{p}^{E,+}$ such that $I_{p}^{I}=\mathcal{R}_{u}^{T}$ and $I_{p}^{E,+}$ is obtained from the SBP-preserving relation, ${ }^{11}$ limited to the nodes not included on the coarse grid $\Omega_{2}$, i.e.

$$
I_{p}^{E,+}= \begin{cases}P_{1}^{-1}\left(I_{r}^{-}\right)^{T} P_{2}, & \text { nodes on } \Omega_{1} \backslash \Omega_{2}, \\ 0, & \text { nodes on } \Omega_{2} .\end{cases}
$$

For simplicity, these prolongation operators will be referred to as SBP-preserving.

The SBP-preserving choice (23) also leads to (13) for first order discretizations. In Section IV.D we will perform a few numerical experiments involving the SBP-preserving prolongation. However, unless otherwise stated, we will use the linear prolongation operator in the following.

\section{IV.B. Second order discretizations}

The second order space discretization of (1) is (2) with

$$
D_{+}=\frac{1}{\Delta x}\left[\begin{array}{ccccccc}
-1 & 1 & & & & & \\
-1 & 1 & & & & & \\
\frac{1}{2} & -2 & \frac{3}{2} & & & & \\
& \ddots & \ddots & \ddots & & & \\
& & \frac{1}{2} & -2 & \frac{3}{2} & & \\
& & & \frac{2}{5} & -\frac{8}{5} & 1 & \frac{1}{5} \\
& & & & 2 & -5 & 3
\end{array}\right], \quad P=\Delta x \cdot \operatorname{diag}\left(\frac{1}{4}, \frac{5}{4}, 1, \ldots, 1, \frac{5}{4}, \frac{1}{4}\right)
$$


Discretizing (2) in time with RK4 we get, as for the first order case, an iterative solver that converges to an approximation of the steady-state solution (5). To accelerate the convergence, we apply (11) with a first order SBP upwind coarse-grid discretization. The residual restriction operator $I_{r}^{+}=L_{2} \mathcal{R}_{u} L_{1}^{-1}$ in this case can be written in closed form as

$$
I_{r}^{+}=\left[\begin{array}{ccccccccccc}
\frac{1}{8} & -\frac{1}{8} & & & & & & & & & \\
& \frac{2}{3} & \frac{1}{3} & & & & & & & \\
& \frac{2}{3^{3}} & \frac{4}{3^{3}} & \frac{4}{3^{2}} & \frac{1}{3} & & & & & \\
\frac{2}{3^{5}} & \frac{4}{3^{5}} & \frac{4}{3^{4}} & \frac{4}{3^{3}} & \frac{4}{3^{2}} & \frac{1}{3} & & & \\
\vdots & \vdots & \cdots & \cdots & \cdots & \ddots & \ddots & & \\
\frac{2}{3^{N-3}} & \frac{4}{3^{N-3}} & \frac{4}{3^{N-4}} & \cdots & \cdots & \cdots & \frac{4}{3^{2}} & \frac{1}{3} & & \\
\frac{1}{4 \cdot 3^{N-3}} & \frac{1}{6 \cdot 3^{N-4}} & \frac{1}{6 \cdot 3^{N-5}} & \cdots & \cdots & \cdots & \frac{1}{6 \cdot 3} & \frac{1}{6} & \frac{5}{8} & \frac{1}{8}
\end{array}\right]
$$

Note that the resulting interpolation is consistent for all nodes except the first one, as for (12).

Unlike the first order case, the residual restriction consists of nonlocal contributions which increase the computational effort. A possible solution to this drawback is to cancel all contributions smaller than a given threshold in absolute value. We have chosen this threshold to be equal to $5 \cdot 10^{-17}$, since we use a double-precision floating-point format where unit roundoff is approximately $10^{-16}$. Figure 7 shows the sparsity patterns for the residual restriction in (25) and its approximated counterpart.
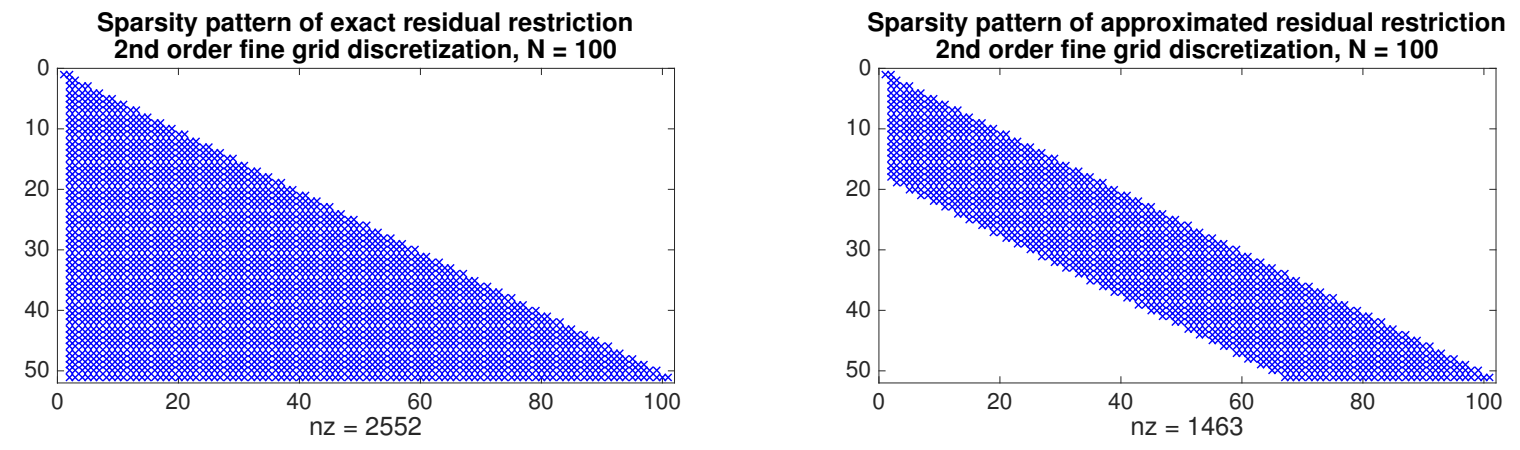

Figure 7. Sparsity patterns of the exact residual restriction operator (25) (left) and its approximated version (right) for the second order fine-grid discretization with $N=100$. The approximated interpolation operator is obtained by cancelling the matrix entries smaller than $5 \cdot 10^{-17}$ in absolute value.

In Figure 8 (left) we compare the convergence of the multigrid algorithm with the exact (25) and the approximate residual restriction operator. The approximate version does not significantly change the convergence results compared to the use of the exact version in (25), and hence it is preferred.

Remark IV.1. As for the single-grid case mentioned in Section II.B, the convergence to steadystate consists of a convective and a damping phase. The residual restriction $I_{r}^{+}=L_{2} \mathcal{R}_{u} L_{1}^{-1}$ makes the convective phase faster and keeps the damping phase almost unaltered. 

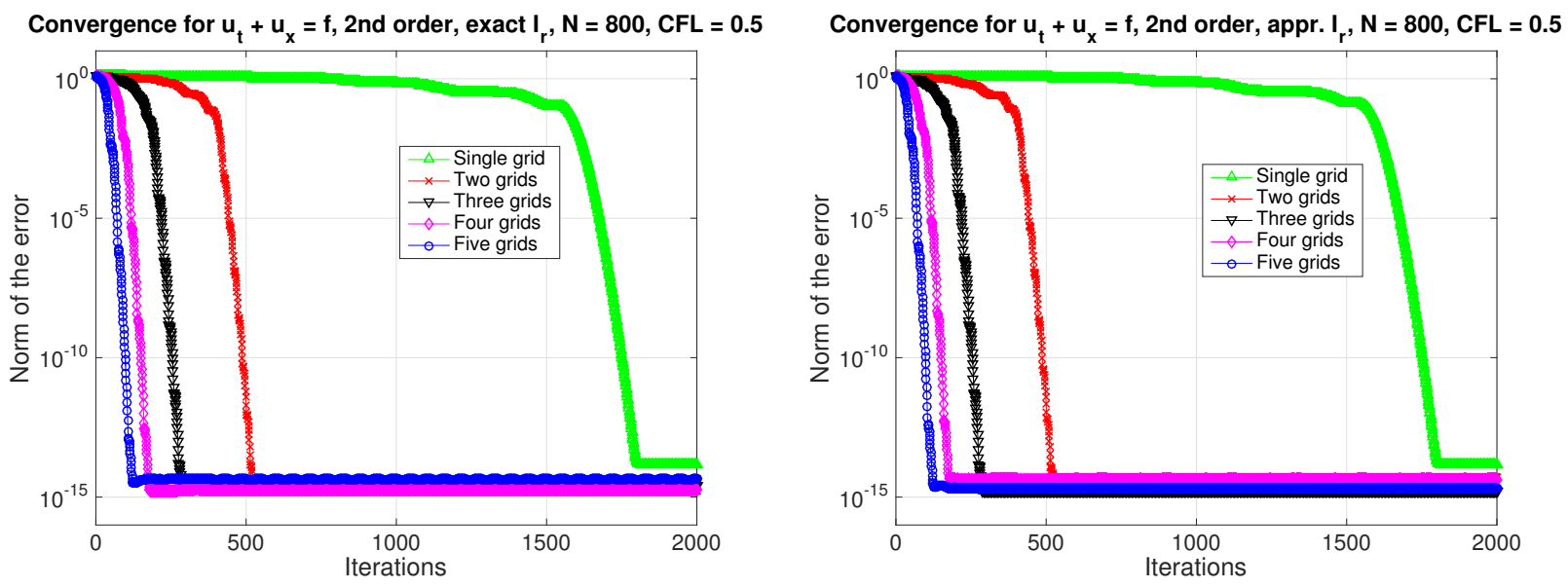

Figure 8. Convergence plots for the multigrid algorithm applied to a second order discretization with $N=800$. The left figure shows the $P$-norm of the error for the exact residual restriction (25), while the convergence for the approximate version is displayed on the right figure. RK4 time marching with $\lambda=0.5$ was used on each grid for wave propagation.

\section{IV.C. Extension to higher orders}

To extend the multigrid algorithm to higher order discretizations, we need to find $I_{r}^{+}$such that $I_{r}^{+} L_{1}=L_{2} \mathcal{R}_{u}$. As for the second order case, it is possible to compute these restriction operators by explicitly inverting the matrix $L_{1}$. However, in general the entries of $I_{r}^{+}$depend on the number of nodes. This makes it unfeasible to exactly represent or store the restriction operator needed for high order discretizations. However, when $N$ is greater than a given $N^{*}$ the approximate version of $I_{r}^{+}$has a stencil independent of the number of nodes. We have therefore computed the approximate version of the residual restriction operators satisfying $I_{r}^{+} L_{1}=L_{2} \mathcal{R}_{u}$ for a $3 \mathrm{rd}$, 4 th, 5 th and 6 th order fine-grid discretization. In Figure 9 the sparsity patterns for the resulting interpolation operators are shown for $N=160$. Once again, the restriction operators are consistent at all nodes except the first one due to the particular structure of the first order upwind operator $D_{+}$in (3).

In Figure 10 we show the convergence plots of the multigrid procedure using the new residual restriction operators $I_{r}^{+}$. Similarly to the first (Figure 4) and second (Figure 8) order discretizations, the new residual restrictions lead to multigrid algorithms with significantly increased wave propagation speed, for all orders of accuracy.

For completeness, Table 1 shows the orders of convergence (computed and expected ${ }^{7}$ ) for different SBP-SAT upwind discretizations of (1). For a $p$ th-order SBP upwind discretization fulfilling Definition II.1, the order of convergence is expected to be at least $\lfloor p / 2\rfloor+1$. Our results show a superconvergence behavior for some discretizations and do not contradict the theory. Note that in practice it is not meaningful to aim for machine precision with multigrid algorithms. It is enough to get an error below the truncation error of the scheme.

\section{IV.D. The SBP-preserving prolongation}

The use of the SBP-preserving prolongation instead of (13) leads to the convergence results shown in Figure 11 for 3rd, 4th, 5th and 6th order discretizations. In terms of iterations to reach convergence, (23) does not yield any substantial improvement (cf. Figure 10). However, the cost in terms of arithmetical operations needed for the SBP-preserving prolongation is relatively high compared to the linear prolongation, due to the sparsity pattern comparable to the one of $I_{r}^{+}$.

Nonetheless, the prolongation in (23) has the advantage of having better stability properties 

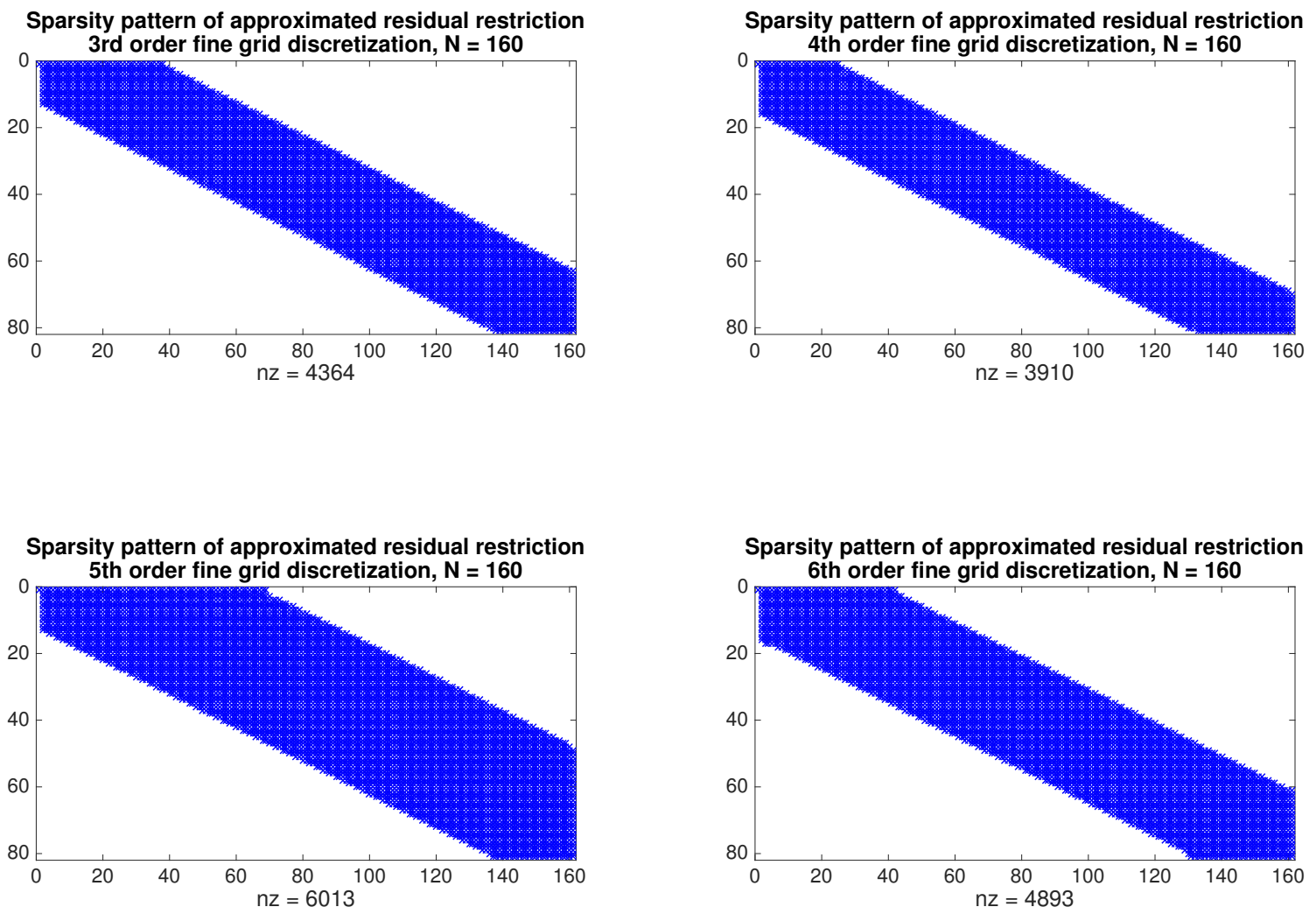

Figure 9. Sparsity patterns of the residual restriction operators satisfying $I_{r}^{+} L_{1}=L_{2} \mathcal{R}_{u}$ for 3 rd (top left), 4th (top right), 5th (bottom left), 6th (bottom right) order discretizations on the fine grid. The approximated interpolation operator is obtained by setting to zero the entries of the matrix smaller of $5 \cdot 10^{-17}$ in absolute value.

compared to the linear prolongation in (13), hence allowing for slightly increased CFL numbers. In Figure 12, the convergence results for the 2nd order discretization with RK4 time-marching and $\lambda=0.6$ using both classes of prolongation operators are shown. In this case the SBP-preserving prolongation prevents overshoots in the transient phase of the two-grid procedure. Despite this benefit, the SBP-preserving prolongation remains costly compared to the linear prolongation. Hence, in the following (13) will be used in the fine grid update step of (11).

\section{Extension to hyperbolic systems of equations}

Consider the linear hyperbolic system of equations

$$
\begin{aligned}
\mathbf{u}_{t}+A \mathbf{u}_{x} & =\mathbf{f}(x), \quad 0<x<1, \quad t>0 \\
\mathbf{u}(x, 0) & =\mathbf{u}^{0}(x), \quad 0<x<1,
\end{aligned}
$$

where $A \in \mathbb{R}^{s \times s}$ is a symmetric matrix with constant coefficients and all the vectors involved have $s$ components. To start with, we associate to (26) the set of characteristic boundary conditions

$$
\begin{aligned}
& X_{+}^{T} \mathbf{u}=\mathbf{g}_{0}, \quad x=0 \\
& X_{-}^{T} \mathbf{u}=\mathbf{g}_{1}, \quad x=1
\end{aligned}
$$



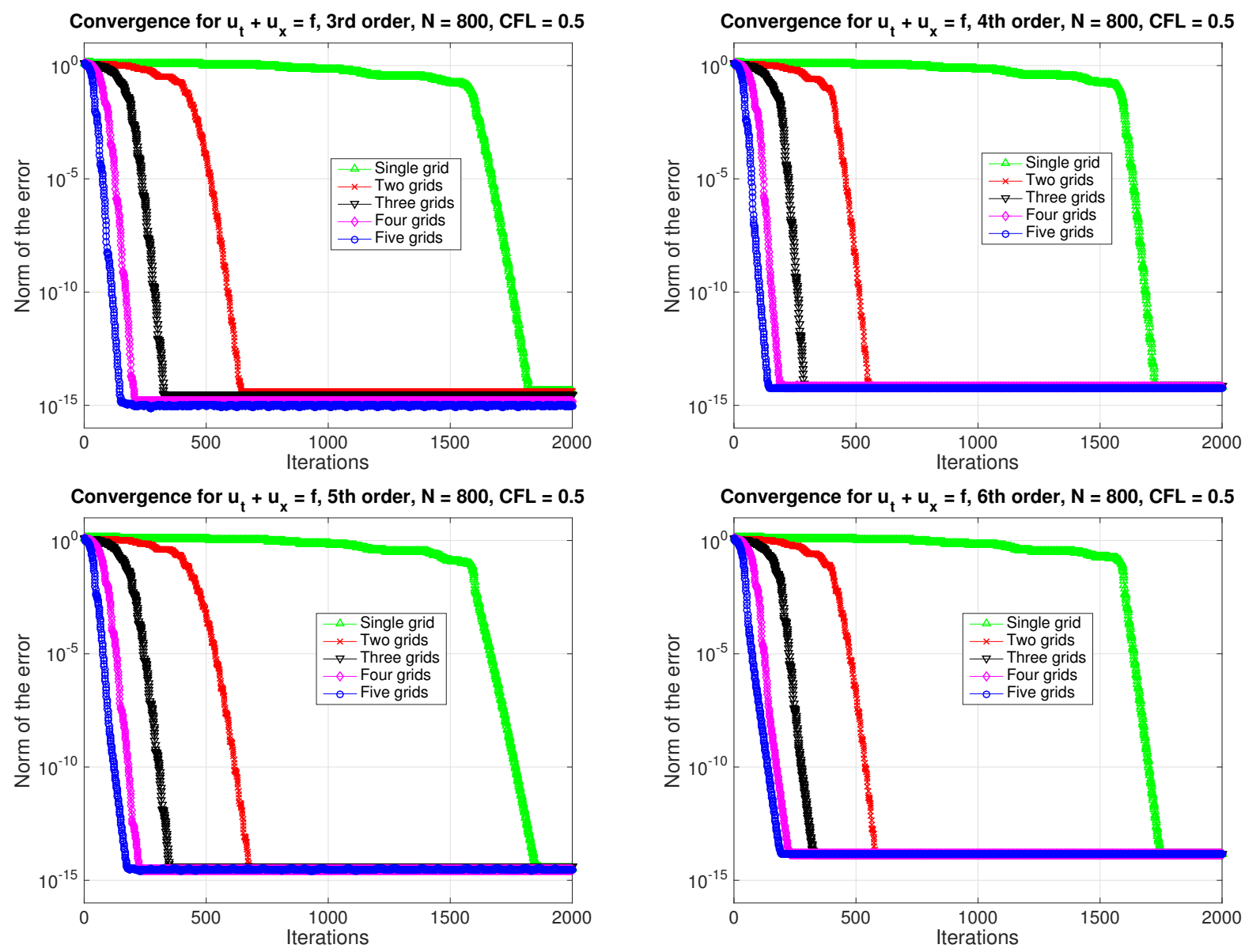

Figure 10. Convergence plots for the multigrid algorithm satisfying $I_{r}^{+} L_{1}=L_{2} \mathcal{R}_{u}$ applied to a 3rd (top left), 4th (top right), 5th (bottom left), 6th (bottom right) order discretization on the fine grid. RK4 time marching with $\lambda=0.5$ was used on each grid for wave propagation.

where $X_{+}$and $X_{-}$indicate the eigenvectors related to the positive and negative eigenvalues of $A$, respectively.

Let $A=X \Lambda X^{T}$ be the eigendecomposition of $A$ : we define $A^{ \pm}=X \Lambda^{ \pm} X^{T}=X_{ \pm} \Lambda^{ \pm} X_{ \pm}^{T}$ such that $A=A^{+}+A^{-}$, where $\Lambda^{+}$and $\Lambda^{-}$are matrices consisting of the positive and negative eigenvalues of $A$. Furthermore, it is convenient to introduce the projection matrices $I_{s}^{ \pm}=X_{ \pm} X_{ \pm}^{T}$ in order to split left- and right-traveling waves. The following relations hold

$$
X=\left[\begin{array}{ll}
X_{+} & X_{-}
\end{array}\right], \quad \Lambda=\left[\begin{array}{cc}
\Lambda^{+} & 0 \\
0 & \Lambda^{-}
\end{array}\right], \quad I_{s}^{+}+I_{s}^{-}=I_{s}, \quad I_{s}^{ \pm} A^{ \pm}=A^{ \pm}, \quad I_{s}^{ \pm} A^{\mp}=0
$$

where $I_{s} \in \mathbb{R}^{s \times s}$ is the identity matrix. The boundary conditions (27) lead to well-posedness of (26), since for $\mathbf{f}=0$ the energy-method gives

$$
\frac{\mathrm{d}}{\mathrm{d} t}\|\mathbf{u}\|_{2}^{2}=\left.\mathbf{u}^{T} A \mathbf{u}\right|_{x=0}-\left.\mathbf{u}^{T} A \mathbf{u}\right|_{x=1}=\mathbf{g}_{0}^{T} \Lambda^{+} \mathbf{g}_{0}-\mathbf{g}_{1}^{T} \Lambda^{-} \mathbf{g}_{1}+\left.\mathbf{u}^{T} A^{-} \mathbf{u}\right|_{x=0}-\left.\mathbf{u}^{T} A^{+} \mathbf{u}\right|_{x=1} .
$$

A semidiscrete SBP-SAT approximation of (26),(27) can be written as ${ }^{7,12}$

$$
\begin{aligned}
\boldsymbol{U}_{t}+\left(A^{+} \otimes D_{+}\right) \boldsymbol{U}+\left(A^{-} \otimes D_{-}\right) \boldsymbol{U}=\boldsymbol{f} & -\left(A^{+} \otimes P^{-1} \mathbf{e}_{0} \mathbf{e}_{0}^{T}\right)\left(\boldsymbol{U}-\widetilde{\boldsymbol{g}}_{0}\right) \\
& +\left(A^{-} \otimes P^{-1} \mathbf{e}_{N} \mathbf{e}_{N}^{T}\right)\left(\boldsymbol{U}-\widetilde{\boldsymbol{g}}_{1}\right),
\end{aligned}
$$




\begin{tabular}{r|r|r|r|r|r|r}
$N \backslash$ Discretization & 1 st & 2 nd & 3 rd & 4 th & 5 th & 6 th \\
\hline 100 & $7.520 \mathrm{e}-2$ & $2.130 \mathrm{e}-2$ & $2.474 \mathrm{e}-3$ & $3.972 \mathrm{e}-4$ & $2.652 \mathrm{e}-4$ & $2.664 \mathrm{e}-4$ \\
200 & $3.785 \mathrm{e}-2$ & $5.563 \mathrm{e}-3$ & $4.134 \mathrm{e}-4$ & $2.303 \mathrm{e}-5$ & $1.412 \mathrm{e}-5$ & $1.430 \mathrm{e}-5$ \\
300 & $2.531 \mathrm{e}-2$ & $2.500 \mathrm{e}-3$ & $1.469 \mathrm{e}-4$ & $4.449 \mathrm{e}-6$ & $2.683 \mathrm{e}-6$ & $2.395 \mathrm{e}-6$ \\
400 & $1.901 \mathrm{e}-2$ & $1.414 \mathrm{e}-3$ & $7.081 \mathrm{e}-5$ & $1.404 \mathrm{e}-6$ & $8.526 \mathrm{e}-7$ & $6.654 \mathrm{e}-7$ \\
500 & $1.522 \mathrm{e}-2$ & $9.072 \mathrm{e}-4$ & $4.027 \mathrm{e}-5$ & $5.789 \mathrm{e}-7$ & $3.569 \mathrm{e}-7$ & $2.455 \mathrm{e}-7$ \\
\hline Order & 0.9965 & 1.9889 & 2.5293 & 3.9703 & 3.9026 & 4.4684 \\
\hline Expected & 1 & 2 & 2 & 3 & 3 & 4
\end{tabular}

Table 1. Truncation errors and orders of convergence for the upwind SBP operators applied to (2). The orders of convergence are computed with the truncation errors of the two finest grids.

where we have introduced the vector $\mathbf{e}_{N}=(0, \ldots, 0,1)^{T}$. Moreover, in $(28)$ the operator $\otimes$ denotes the Kronecker product defined by

$$
X \otimes Y=\left[\begin{array}{ccc}
x_{11} Y & \cdots & x_{1 n} Y \\
\vdots & \ddots & \vdots \\
x_{m 1} Y & \cdots & x_{m n} Y
\end{array}\right] \in \mathbb{R}^{m r \times n k}, \quad X=\left\{a_{i j}\right\} \in \mathbb{R}^{m \times n}, \quad Y \in \mathbb{R}^{r \times k}
$$

The discrete operator $\left(A_{+} \otimes D_{+}\right)+\left(A_{-} \otimes D_{-}\right)$in $(28)$ can be recast to highlight the relation between the SBP upwind operators $\left(D_{+}, D_{-}\right)$and the SBP operators based on centered difference methods $\left(D_{c}\right)^{8}$ as

$$
\begin{aligned}
\left(A_{+} \otimes D_{+}\right)+\left(A_{-} \otimes D_{-}\right) & =\left(A \otimes \frac{D_{+}+D_{-}}{2}\right)+\left(\left(A^{+}-A^{-}\right) \otimes \frac{D_{+}-D_{-}}{2}\right) \\
& =\left(A \otimes D_{c}\right)+\left(|A| \otimes P^{-1} S\right) .
\end{aligned}
$$

In (29), $|A|=A^{+}-A^{-}$has non-negative eigenvalues and $S=\frac{1}{2}\left(Q_{+}-Q_{-}\right)=\frac{1}{2}\left(Q_{+}+Q_{+}^{T}\right)$ is a positive semi-definite matrix which can be seen as an artificial dissipation term. ${ }^{7,13}$ As a consequence, the stability of (28) can be proved $^{6}$ in the same way as for the usual SBP operators.

\section{V.A. Modifications of the multigrid procedure for systems of equations}

To apply the multigrid algorithm to hyperbolic systems, we need to recast the semi-discrete formulation (28) in compact form, as we did for the scalar problem (2). By introducing $L_{1}^{+}=D_{+}+P^{-1} \mathbf{e}_{0} \mathbf{e}_{0}^{T}$ and $L_{1}^{-}=D_{-}-P^{-1} \mathbf{e}_{N} \mathbf{e}_{N}^{T}$, we can write $L_{1}=\left(A_{+} \otimes L_{1}^{+}\right)+\left(A_{-} \otimes L_{1}^{-}\right)$and $(28)$ becomes

$$
\boldsymbol{U}_{t}+L_{1} \boldsymbol{U}=\boldsymbol{f}+\left(A^{+} \otimes P^{-1} \mathbf{e}_{0} \mathbf{e}_{0}^{T}\right) \widetilde{\boldsymbol{g}}_{0}-\left(A^{-} \otimes P^{-1} \mathbf{e}_{N} \mathbf{e}_{N}^{T}\right) \widetilde{\boldsymbol{g}}_{1}=: \mathbf{F}_{1}
$$

In a similar manner as for the scalar case, convergence to steady-state can now be accelerated by using the following two-grid algorithm:

Fine grid smoothing: $\quad \overline{\boldsymbol{U}}^{(1)}=S_{1} \boldsymbol{U}^{n}+\left(I_{1}-S_{1}\right) L_{1}^{-1} \boldsymbol{F}_{1}$,

\begin{tabular}{ll} 
Fine grid residual: & $\overline{\boldsymbol{r}}^{(1)}=L_{1} \overline{\boldsymbol{U}}^{(1)}-\boldsymbol{F}_{1}$, \\
Solution injection: & $\boldsymbol{U}^{(2)}=\mathcal{R}_{u}^{s} \overline{\boldsymbol{U}}^{(1)}$, \\
\hline Coarse grid residual: & $\boldsymbol{F}_{2}=L_{2} \boldsymbol{U}^{(2)}-I_{r} \overline{\boldsymbol{r}}^{(1)}$,
\end{tabular}



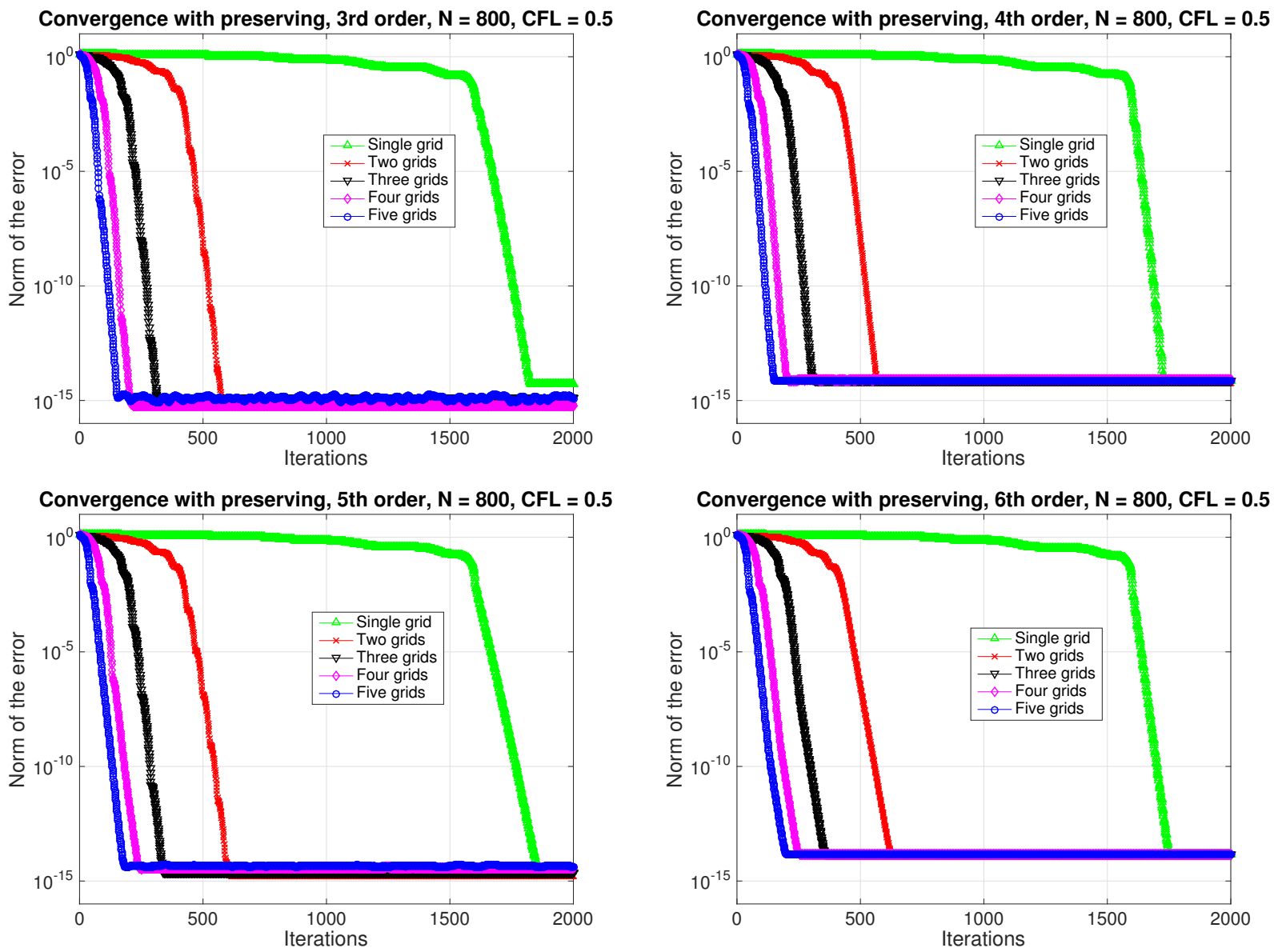

Figure 11. Convergence plots for the multigrid algorithm satisfying $I_{r}^{+} L_{1}=L_{2} \mathcal{R}_{u}$ applied to a 3 rd (top left), 4th (top right), 5th (bottom left), 6th (bottom right) order discretization on the fine grid. The SBP-preserving prolongation (23) was used in the fine grid update step and RK4 time marching with $\lambda=0.5$ was used on each grid for wave propagation.

Coarse grid evolution: $\quad \overline{\boldsymbol{U}}^{(2)}=S_{2} \boldsymbol{U}^{(2)}+\left(I_{2}-S_{2}\right) L_{2}^{-1} \boldsymbol{F}_{2}$,

Fine grid update: $\quad \boldsymbol{U}^{n+1}=\overline{\boldsymbol{U}}^{(1)}+\left(I_{s} \otimes I_{p}^{I}\right)\left(\overline{\boldsymbol{U}}^{(2)}-\mathcal{R}_{u}^{s} \overline{\boldsymbol{U}}^{(1)}\right)$

$$
+\left[\left(I_{s}^{+} \otimes I_{p}^{E,+}\right)+\left(I_{s}^{-} \otimes I_{p}^{E,-}\right)\right]\left(\bar{U}^{(2)}-\mathcal{R}_{u}^{s} \boldsymbol{U}^{n}\right),
$$

where, as in (11), $L_{2}=\left(A_{+} \otimes L_{2}^{+}\right)+\left(A_{-} \otimes L_{2}^{-}\right)$indicates a coarse-grid counterpart of $L_{1}$ and $S_{i}$ is a matrix representing a time-marching procedure on $\Omega_{i}, i=1,2$. We have also introduced the restriction operators

$$
\mathcal{R}_{u}^{s}=I_{s} \otimes \mathcal{R}_{u}, \quad I_{r}=\left(I_{s}^{+} \otimes I_{r}^{+}\right)+\left(I_{s}^{-} \otimes I_{r}^{-}\right)
$$

which lead to the following

Lemma V.1. The operators in (32) yield $I_{r} L_{1}=L_{2} \mathcal{R}_{u}^{s}$ for the characteristic boundary conditions (27).

Proof. The result can be proved by computing $I_{r} L_{1}$ as

$$
I_{r} L_{1}=\left[\left(I_{s}^{+} \otimes I_{r}^{+}\right)+\left(I_{s}^{-} \otimes I_{r}^{-}\right)\right]\left[\left(A^{+} \otimes L_{1}^{+}\right)+\left(A^{-} \otimes L_{1}^{-}\right)\right]
$$



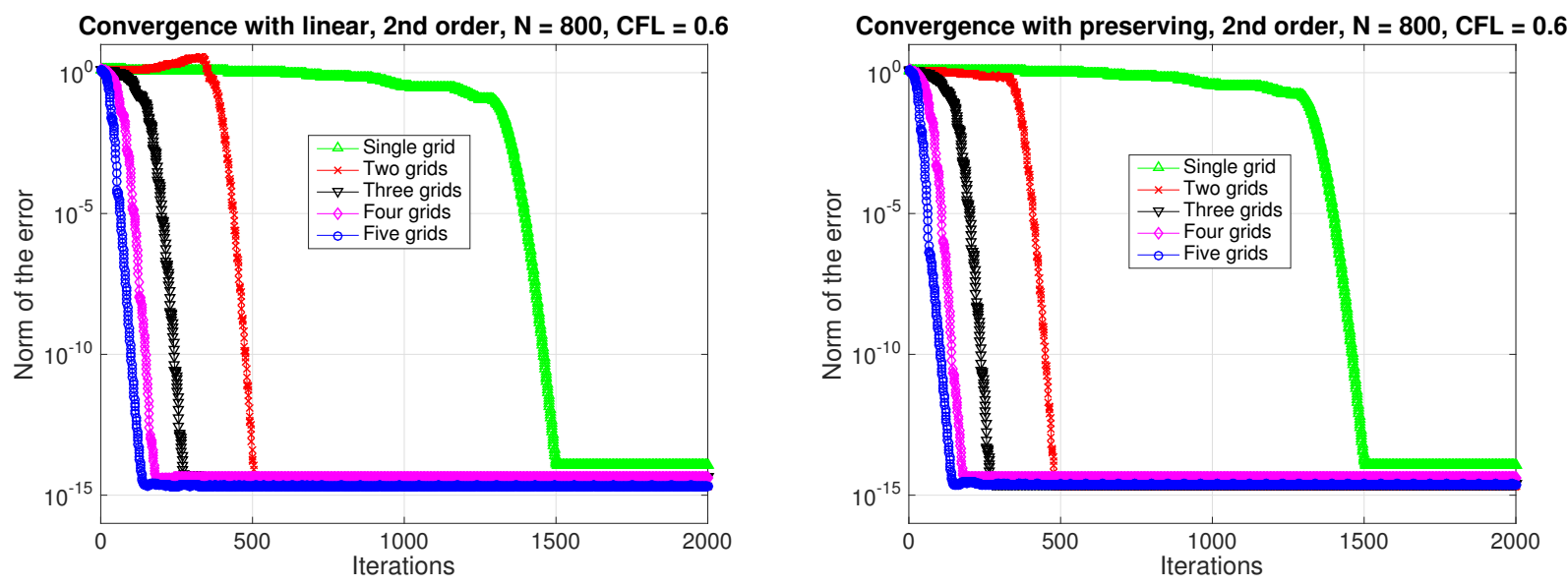

Figure 12. Convergence plots for the multigrid algorithm satisfying $I_{r}^{+} L_{1}=L_{2} \mathcal{R}_{u}$ applied to a 2 nd order discretization on the fine grid. The linear (left) and the SBP-preserving prolongation (right) were used in the fine grid update step. Moreover, RK4 time marching with $\lambda=0.6$ was used on each grid for wave propagation.

$$
\begin{aligned}
& =(\underbrace{I_{s}^{+} A^{+}}_{=A^{+}} \otimes I_{r}^{+} L_{1}^{+})+(\underbrace{I_{s}^{+} A^{-}}_{=0} \otimes I_{r}^{+} L_{1}^{-})+(\underbrace{I_{s}^{-} A^{+}}_{=0} \otimes I_{r}^{-} L_{1}^{+})+(\underbrace{I_{s}^{-} A^{-}}_{=A^{-}} \otimes I_{r}^{-} L_{1}^{-}) \\
& =\left(A^{+} \otimes I_{r}^{+} L_{1}^{+}\right)+\left(A^{-} \otimes I_{r}^{-} L_{1}^{-}\right) .
\end{aligned}
$$

Since $I_{r}^{+}$and $L_{1}^{+}$are the same matrices as in the scalar problem, their product fulfills $I_{r}^{+} L_{1}^{+}=L_{2}^{+} \mathcal{R}_{u}$ with $L_{2}^{+}=D_{+, 2}+P_{2}^{-1} \mathbf{e}_{0,2} \mathbf{e}_{0,2}^{T}$. Similarly, $I_{r}^{-} L_{1}^{-}=L_{2}^{-} \mathcal{R}_{u}$, where $L_{2}^{-}=D_{-, 2}-P_{2}^{-1} \mathbf{e}_{N, 2} \mathbf{e}_{N, 2}^{T}$, and hence

$$
I_{r} L_{1}=\left(A^{+} \otimes L_{2}^{+} \mathcal{R}_{u}\right)+\left(A^{-} \otimes L_{2}^{-} \mathcal{R}_{u}\right)=\left[\left(A^{+} \otimes L_{2}^{+}\right)+\left(A^{-} \otimes L_{2}^{-}\right)\right]\left(I_{s} \otimes \mathcal{R}_{u}\right)=L_{2} \mathcal{R}_{u}^{s} .
$$

Remark V.2. The residual restriction $I_{r}$ in (32), which consists of the projection matrices $I_{s}^{ \pm}$, has the advantage of making the mixed contributions in $I_{r} L_{1}$ disappear.

Lemma V.1 implies that the operators $I_{r}^{+}$and $I_{r}^{-}$, previously computed for the scalar case, can be used to write a residual restriction $I_{r}$ which verifies the assumption (14). This results suggests that the algorithm (31) behaves similarly to (11) for problems with characteristic boundary conditions such as $(26),(27)$.

\section{V.B. Numerical results for characteristic boundary conditions}

To test the algorithm (31), we study the linearized one dimensional symmetrized form of the compressible Euler equations ${ }^{14}$

$$
\begin{aligned}
\mathbf{U}_{t}+A \mathbf{U}_{x} & =\mathbf{F}, \quad 0<x<1, \quad t>0 \\
\mathbf{U}(x, 0) & =\mathbf{f}, \quad 0<x<1,
\end{aligned}
$$

In (33) we have

$$
\mathbf{U}=\left[\frac{\bar{c}}{\sqrt{\gamma} \bar{\rho}} \rho, u, \frac{1}{\bar{c} \sqrt{\gamma(\gamma-1)}} \theta\right]^{T}, \quad A=\left[\begin{array}{ccc}
\bar{u} & \frac{\bar{c}}{\sqrt{\gamma}} & 0 \\
\overline{\bar{c}} & \bar{u} & \sqrt{\frac{\gamma-1}{\gamma}} \bar{c} \\
0 & \sqrt{\frac{\gamma-1}{\gamma}} \bar{c} & \bar{u}
\end{array}\right]
$$


with $\bar{u}=1, \bar{c}=2, \bar{\rho}=1$ and $\gamma=1.4$. The variables $\rho, u$ and $\theta$ are the density, velocity and temperature perturbations of the fluid, respectively. The characteristic boundary conditions (27) become

$$
\begin{aligned}
\frac{\bar{c}}{\bar{\rho}} \rho-\frac{\theta}{\bar{c}(\gamma-1)}=g_{01}, & x=0, \\
\overline{\bar{c}} \rho+\gamma u+\frac{\theta}{\overline{\bar{c}}}=g_{02}, & x=0, \\
\overline{\bar{c}} \rho-\gamma u+\frac{\theta}{\overline{\bar{c}}}=g_{1}, & x=1 .
\end{aligned}
$$

Consider the manufactured solution

$$
\rho=e^{-\nu \cos ^{2}(\xi \pi x)}, \quad u=\cos (\xi \pi x), \quad \theta=e^{-\nu \sin ^{2}(\xi \pi x)}
$$

with $\nu=0.1, \xi=2$ and a random initial data compatible with the boundary conditions. The multigrid convergence results for the 1st, 2nd, 3rd, 4th, 5th and 6th order upwind discretizations of (33), (34) are shown in Figure 13. As expected, recursively applying the algorithm in (31) makes wave propagation faster. Note that for the two-grid algorithm applied to the 2nd order discretization overshoots occur. As mentioned in Section IV.D, this side effect can be eliminated by using the SBP-preserving prolongation (23) in the fine-grid update step of (31).

\section{V.C. Numerical results for other boundary conditions}

Other sets of boundary conditions can also lead to a well-posed problem. Consider a rotation of the matrix $A=Y \Omega Y^{T}$ using

$$
Y=\left[\begin{array}{ccc}
1 & 0 & 0 \\
\overline{\bar{c}} & 1 & 0 \\
\bar{u} \sqrt{\gamma} & \sqrt{\frac{\gamma-1}{\gamma}} \frac{\bar{u} \gamma \bar{c}}{\bar{u}^{2} \gamma-\bar{c}^{2}} & 1
\end{array}\right], \quad \Omega=\operatorname{diag}\left(\bar{u}, \frac{\bar{u}^{2} \gamma-\bar{c}^{2}}{\bar{u} \gamma}, \frac{\bar{u} \gamma\left(\bar{u}^{2}-\bar{c}^{2}\right)}{\bar{u}^{2} \gamma-\bar{c}^{2}}\right) .
$$

Since we consider a subsonic flow $(\bar{u}<\bar{c})$, two boundary conditions must be imposed at $x=0$ while the remaining boundary condition is set at $x=1$. In particular,

$$
\begin{aligned}
& Y_{+}^{T} \mathbf{u}=\mathbf{g}_{0}, \quad x=0 \\
& Y_{-}^{T} \mathbf{u}=\mathbf{g}_{1}, \quad x=1
\end{aligned}
$$

lead to the boundary conditions

$$
\begin{aligned}
\frac{\rho}{\bar{\rho}}+\frac{u}{\bar{u}} & =g_{0,1}, & x=0, \\
\theta & =g_{0,2}, & x=0, \\
u+\frac{\bar{u}}{\bar{u}^{2} \gamma-\bar{c}^{2}} \theta & =g_{2}, & x=1,
\end{aligned}
$$

and a well-posed problem. The semi-discrete formulation

$$
\begin{aligned}
\boldsymbol{U}_{t}+\left(A^{+} \otimes D_{+}\right) \boldsymbol{U}+\left(A^{-} \otimes D_{-}\right) \boldsymbol{U}=\boldsymbol{f} & -\left(Y_{+} \Omega Y_{+}^{T} \otimes P^{-1} \mathbf{e}_{0} \mathbf{e}_{0}^{T}\right)\left(\boldsymbol{U}-\widetilde{\boldsymbol{g}}_{0}\right) \\
& +\left(Y_{-} \Omega Y_{-}^{T} \otimes P^{-1} \mathbf{e}_{N} \mathbf{e}_{N}^{T}\right)\left(\boldsymbol{U}-\widetilde{\boldsymbol{g}}_{1}\right),
\end{aligned}
$$

can be shown to be stable ${ }^{12}$ (it can be rewritten in terms of central difference operators $D_{c}$ due to $(29))$.

Recursively applying the two-grid algorithm (31) to (37) leads to the convergence results in Figure 14. Since $L_{1}=\left(A_{+} \otimes D_{+}\right)+\left(A_{-} \otimes D_{-}\right)+\left(Y_{+} \Omega Y_{+}^{T} \otimes P^{-1} \mathbf{e}_{0} \mathbf{e}_{0}^{T}\right)-\left(Y_{-} \Omega Y_{-}^{T} \otimes P^{-1} \mathbf{e}_{N} \mathbf{e}_{N}^{T}\right)$, Lemma V.1 does not hold in this case, and $I_{r}$ in (32) fulfills $I_{r} L_{1}=L_{2} \mathcal{R}_{u}^{s}$ only at the interior nodes. Despite this fact, the multigrid procedure leads to faster convergence for all the order of accuracy. 
The convergence to steady-state is slower compared to the one with the characteristic boundary conditions in (34) (cf. Figure 13). In particular, the damping phase seems to be dominating over the wave propagation for both the single- and multi-grid iterative methods.

More generally, the following set of boundary conditions

$$
\begin{aligned}
& Y_{+}^{T} \mathbf{u}-R_{0} Y_{-}^{T} \mathbf{u}=\mathbf{g}_{0}, \quad x=0 \\
& Y_{-}^{T} \mathbf{u}-R_{1} Y_{+}^{T} \mathbf{u}=\mathbf{g}_{1}, \quad x=1
\end{aligned}
$$

lead to the well-posedness of (33) if

$$
\Omega^{-}+R_{0}^{T} \Omega^{+} R_{0}<0, \quad \Omega^{+}+R_{1}^{T} \Omega^{-} R_{1}>0 .
$$

If (39) holds, the semi-discrete SBP-SAT approximation of (33), (38) is stable. ${ }^{12}$ As an example, the matrices

$$
R_{0}=\frac{1}{\sqrt{2}}\left[\begin{array}{l}
1 \\
1
\end{array}\right] \quad R_{1}=\left[\begin{array}{ll}
1 / 3 & 1 / 2
\end{array}\right]
$$

verify (39) for the rotation (35) and hence can be used in (38) to produce stable boundary conditions for (33). The convergence results for the multigrid algorithm in this case are shown in Figure 15. Once again, faster convergence to steady-state is achieved proportionally to the number of grids used, but the results are slower compared to the one with $R_{0}=R_{1}=0$ as in (36) (cf. Figure 14).

\section{Conclusions and future work}

In this paper, we have replicated and extended the first order accurate TVD-MG scheme ${ }^{4,5}$ to upwind Summation-By-Parts (SBP) based high-order accurate finite difference methods ${ }^{6,7}$ for linear hyperbolic problems. We have reinterpreted the upwind biased interpolation from a matrixvector perspective, leading to more general residual restriction operators. These new operators allowed us to complement the wave propagation for high-order discretizations with the TVD-MG scheme for first order approximations on coarse grids.

Furthermore, we have shown that the restriction operators needed to accelerate wave propagation for the linear advection equation can be used to improve the procedure for hyperbolic systems. For characteristic boundary conditions, the new multigrid scheme leads to wave propagation with increasing speed as the number of grids increaseas. For other stable boundary conditions, the effect of this algorithm is to increase the convergence rate.

We envision that the residual restriction operators derived in this paper can be used to extend the multigrid scheme to multiple dimensions. Modifications of the current algorithm may be required, since the crucial assumption $I_{r} L_{1}=L_{2} \mathcal{R}_{u}$ can only be fulfilled by a residual restriction $I_{r}$ obtained by inverting the fine-grid operator $L_{1}$. This operation is neither possible, due to the increased dimension of the system, nor useful, since it would provide restriction operators only for a particular linear combination of discrete operators on coordinate directions, e.g. $L_{1}=\alpha L_{1, x}+\beta L_{1, y}$.

\section{Acknowledgments}

This work was supported by VINNOVA, the Swedish Governmental Agency for Innovation Systems, under contract number 2013-01209. 

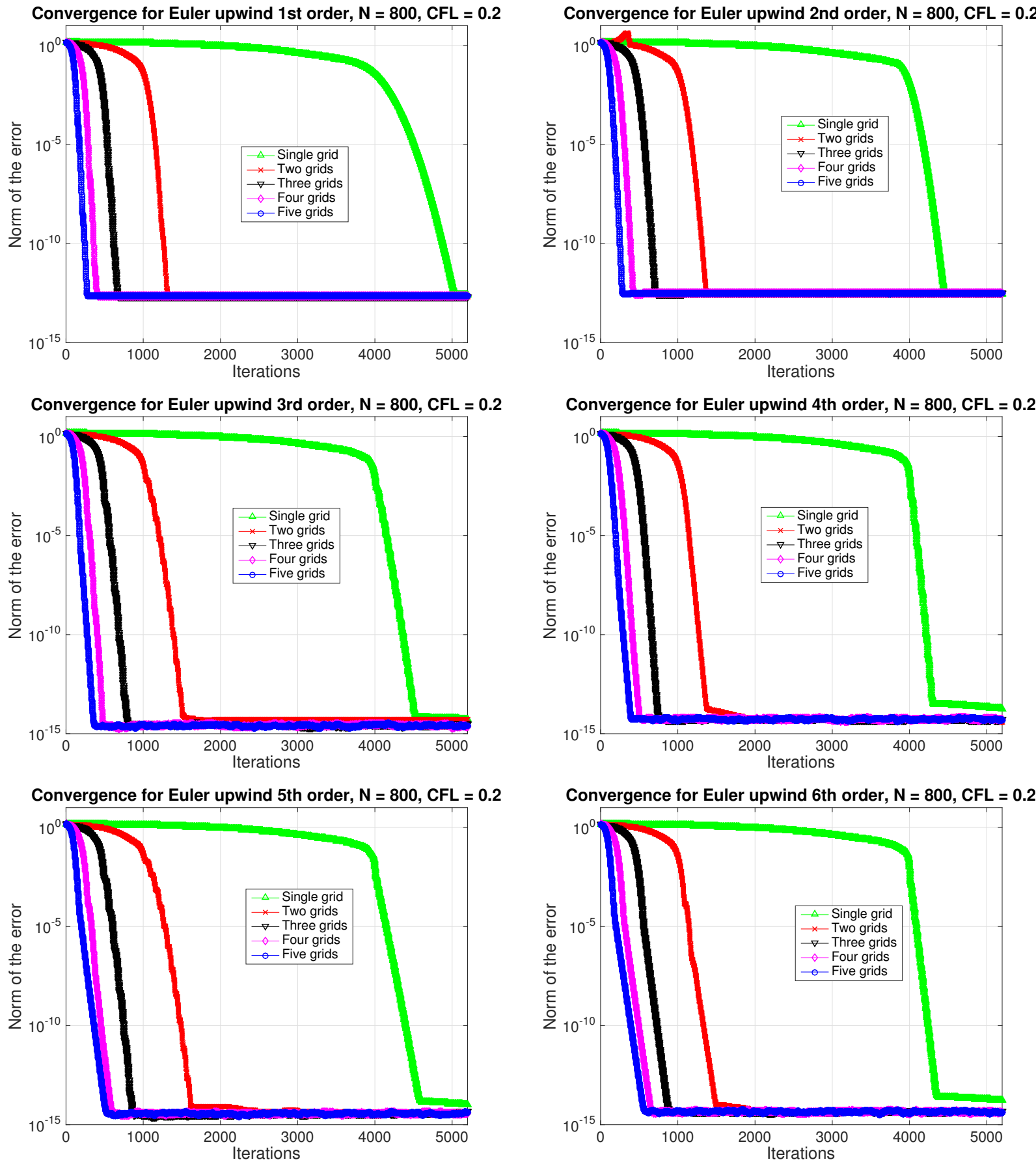

Figure 13. Convergence plots for the multigrid algorithm (31) applied to the linearized one dimensional symmetrized form of the compressible Euler equations (33) with characteristic boundary conditions (34). 

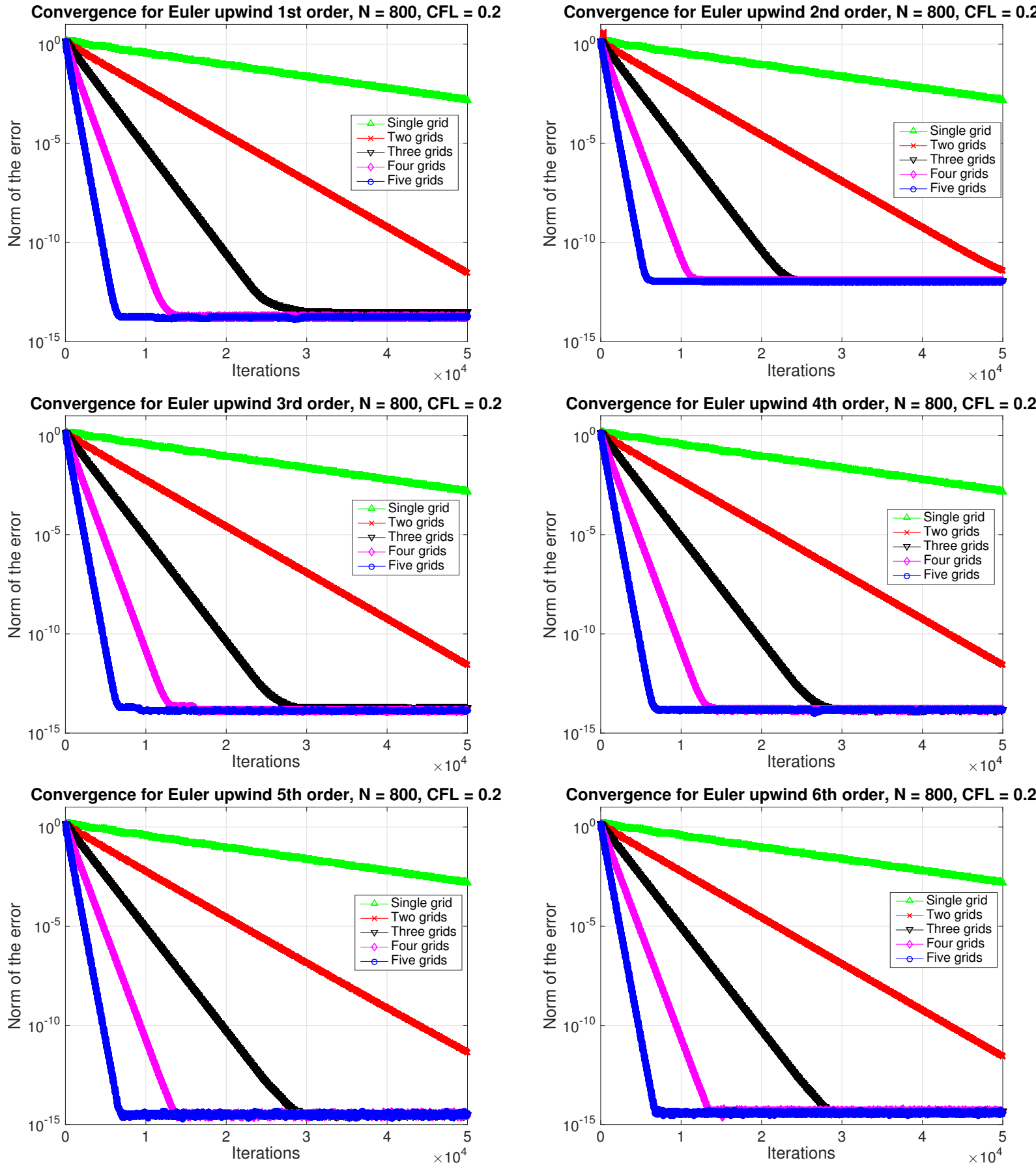

Figure 14. Convergence plots for the multigrid algorithm (31) applied to the linearized one dimensional symmetrized form of the compressible Euler equations (33) with (36). 

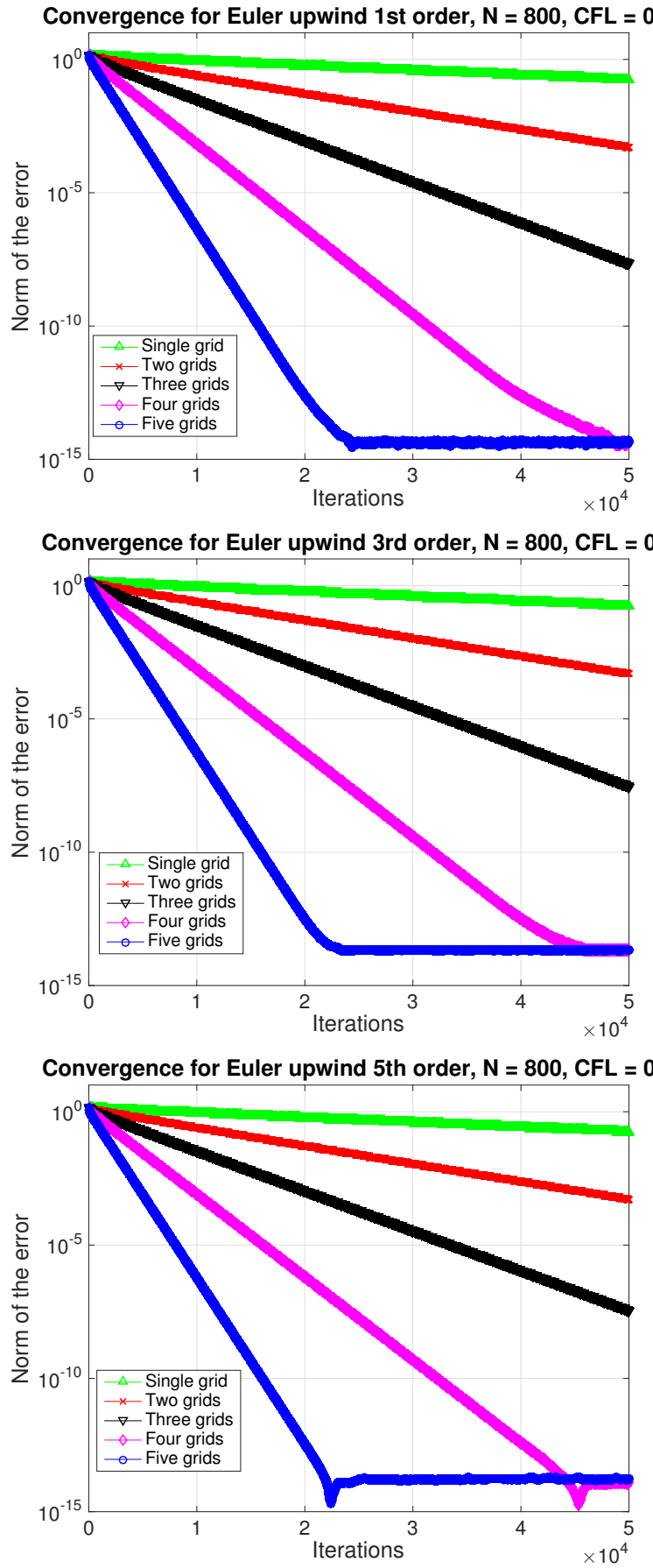
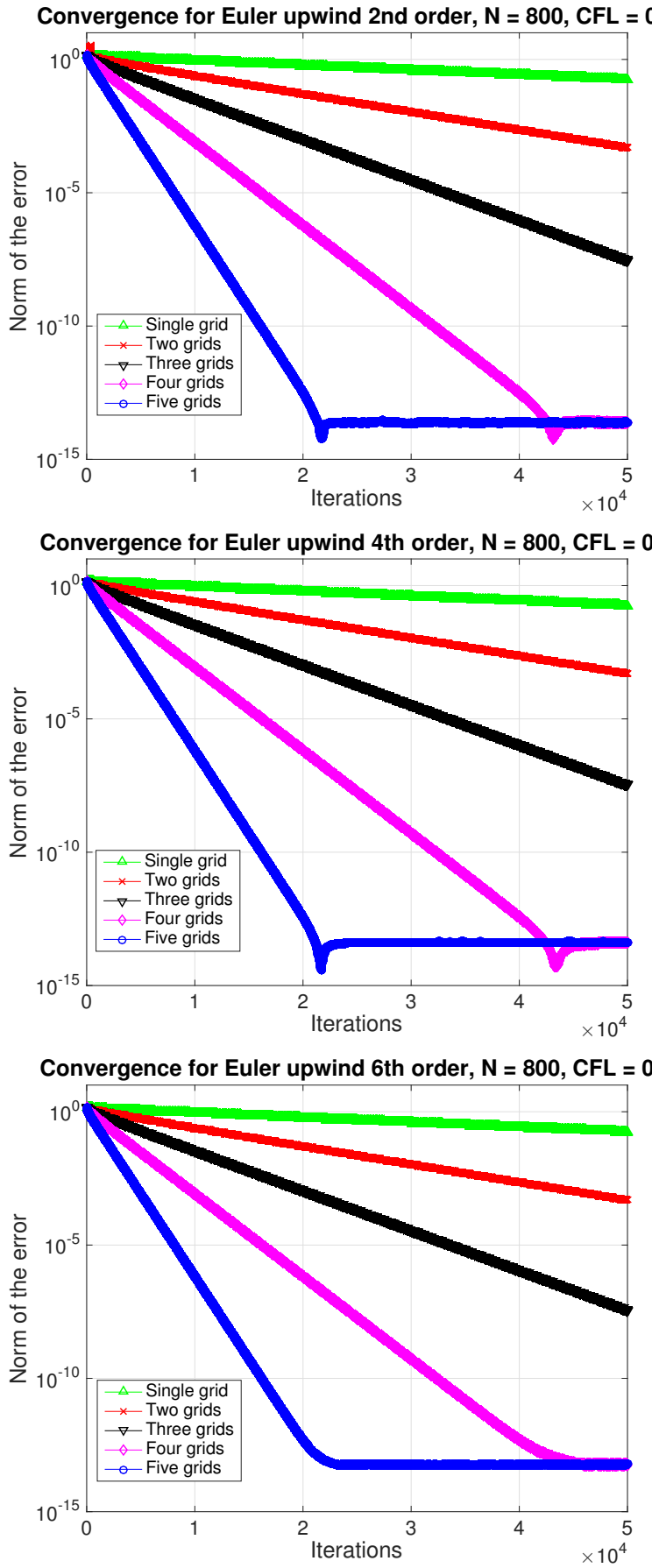

Figure 15. Convergence plots for the multigrid algorithm (31) applied to the linearized one dimensional symmetrized form of the compressible Euler equations (33) with (38), (40). 


\section{References}

${ }^{1}$ R. P. Fedorenko, Iterative methods for elliptic difference equations. Russian Mathematical Surveys. 28, 129-195 (1973)

${ }^{2}$ W. Hackbusch, Multi-Grid Methods and Applications. Springer. (1985)

${ }^{3}$ S. F. McCormick, Multigrid methods. SIAM. (1987)

${ }^{4}$ J. W. L. Wan, A. Jameson, Monotonicity preserving multigrid time stepping schemes for conservation laws. Computing and Visualization in Science. 11, 41-58 (2008)

${ }^{5}$ S. Amarala, J. W. L. Wan, Multigrid methods for systems of hyperbolic conservation laws. Multiscale Modeling \& Simulations. 11, 586-614 (2013)

${ }^{6}$ M. Svärd, M., J. Nordström, Review of Summation-By-Parts Schemes for Initial-Boundary-Value Problems. Journal of Computational Physics. 268, 17-38 (2014)

${ }^{7}$ K. Mattsson, Diagonal-norm upwind SBP operators. Journal of Computational Physics. 335, 283-310 (2017)

${ }^{8}$ D. C. Del Rey, J. E. Hicken, D. W. Zingg, Review of summation-by-parts operators with simultaneous approximation terms for the numerical solution of partial differential equations. Computers \& Fluids. 95, 171-196 (2014)

${ }^{9}$ A. A. Ruggiu, J. Nordström, On pseudo-spectral time discretizations in summation-by-parts form. Journal of Computational Physics. 360, 192-201 (2018)

${ }^{10}$ J. Nordström, T. Lundquist, Summation-by-parts in time. Journal of Computational Physics. 251, 487-499 (2013)

${ }^{11}$ A. A. Ruggiu, J. Nordström, A New Multigrid Formulation for High Order Finite Difference Methods on Summation-by-Parts Form. Journal of Computational Physics. 359, 216-238 (2018)

${ }^{12}$ J. Nordström, A Roadmap to Well Posed and Stable Problems in Computational Physics. Journal of Scientific Computing. 71, 365-385 (2017)

${ }^{13}$ K. Mattsson, M. Svärd, J. Nordström, Stable and Accurate Artificial Dissipation. Journal of Scientific Computing. 21, 57-79 (2004)

${ }^{14}$ S. Abarbanel, D. Gottlieb, Optimal time splitting for two- and three-dimensional Navier-Stokes equations with mixed derivatives. Journal of Computational Physics. 41, 1-43 (1981) 